\title{
Wrinkle-to-fold transition in soft layers under equi-biaxial strain: A weakly nonlinear analysis
}

\author{
P. Ciarletta ${ }^{\mathrm{a}, \mathrm{b}, *}$ \\ ${ }^{a}$ CNRS and Sorbonne Universités, UPMC Univ Paris 06, UMR 7190, Institut Jean le Rond d'Alembert, 4 place Jussieu case 162, 75005 Paris, France \\ ${ }^{\mathrm{b}}$ MOX - Politecnico di Milano and Fondazione CEN, piazza Leonardo da Vinci 32, 20133 Milano, Italy
}

\begin{abstract}
A B S T R A C T
Soft materials can experience a mechanical instability when subjected to a finite compression, developing wrinkles which may eventually evolve into folds or creases. The possibility to control the wrinkling network morphology has recently found several applications in many developing fields, such as scaffolds for biomaterials, stretchable electronics and surface microfabrication. Albeit much is known of the pattern initiation at the linear stability order, the nonlinear effects driving the pattern selection in soft materials are still unknown. This work aims at investigating the nature of the elastic bifurcation undertaken by a growing soft layer subjected to a equi-biaxial strain. Considering a skin effect at the free surface, the instability thresholds are found to be controlled by a characteristic length, defined by the ratio between capillary energy and bulk elasticity. For the first time, a weakly nonlinear analysis of the wrinkling instability is performed here using the multiple-scale perturbation method applied to the incremental theory in finite elasticity. The Ginzburg-Landau equations are derived for different superposing linear modes. This study proves that a subcritical pitchfork bifurcation drives the observed wrinkle-to-fold transition in swelling gels experiments, favoring the emer-gence of hexagonal creased patterns, albeit quasi-hexagonal patterns might later emerge because of an expected symmetry break. Moreover, if the surface energy is somewhat comparable to the bulk elastic energy, it has the same stabilizing effect as for fluid instabilities, driving the formation of stable wrinkles, as observed in elastic bi-layered materials.
\end{abstract}

Keywords:

Elastic instability

Wrinkling

Ginzburg-Landau equations

Pattern selection

Multiple-scale analysis

\section{Introduction}

Soft elastic materials can experience a mechanical instability when subjected to a finite compression, developing surface undulations, i.e. wrinkles, which may eventually evolve into folds or creases. From the theoretical viewpoint, the wrinkling of an incompressible elastic half-space was studied by Biot (1963), who proved that all surface modes become unstable when a critical compressive strain is imposed. Because the vanishing velocity of the Rayleigh surface wave triggers such a threshold value, this phenomenon is often referred to as a surface instability. This instability lacks a characteristic length-

\footnotetext{
* Correspondence address: CNRS and Sorbonne Universités, UPMC Univ Paris 06, UMR 7190, Institut Jean le Rond d'Alembert, 4 place Jussieu case 162, 75005 Paris, France.

E-mail address: ciarletta@dalembert.upmc.fr
} 
scale, and a peculiar behavior arises with the fastest growing mode having infinite wavenumber. Not only the wavelength is undetermined, but the instability modes are also found to be energetically unstable (Cao and Hutchinson, 2012). In the real world, such singularities are regularized by the presence of any microstructural length, e.g. a skin effect at the free surface, and complex surface patterns emerge for different applied strains, as shown in Fig. 1.

Since the pioneering works of Tanaka and coworkers on the swelling instability in polymeric gels (Tanaka et al., 1987, 1992), many experimental studies have been recently focused on the pattern formation and dynamics in soft layers. In fact, the possibility to control the wrinkling network morphology has found several applications in many developing fields, such as scaffolds for biomaterials (Kim et al., 2009), stretchable electronics (Rogers et al., 2010), and surface micro-fabrication (Breid and Crosby, 2011). Such a mechanical instability has also been found to trigger morphogenesis in living materials, from soft tissues (Li et al., 2012) to solid tumors (Ciarletta, 2013).

Albeit much is known of the pattern initiation at the linear stability order, the nonlinear behavior driving the wrinkle-tofold transition in soft materials under equi-biaxial strain has only been partially investigated. Numerical studies have evidenced that sinusoidal wrinkles can continuously evolve into stable folded patterns if a compliant substrate is considered (Audoly and Boudaoud, 2008), while the formation of a crease (i.e. the sudden nucleation of a localized fold) is energetically favored in compressed soft layers (Hong et al., 2009). Nevertheless, as creases have been shown to result from a scale-free,
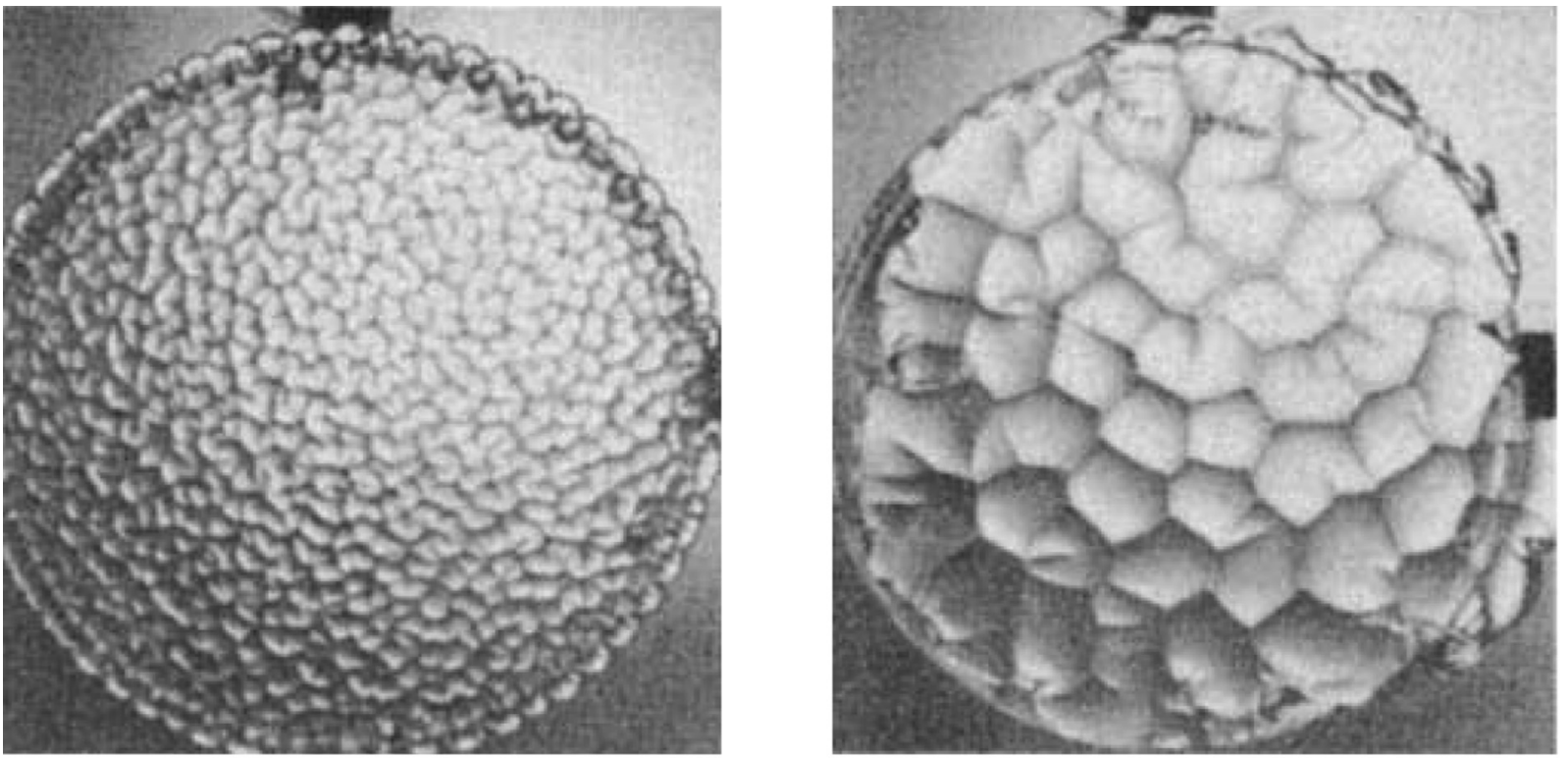

A

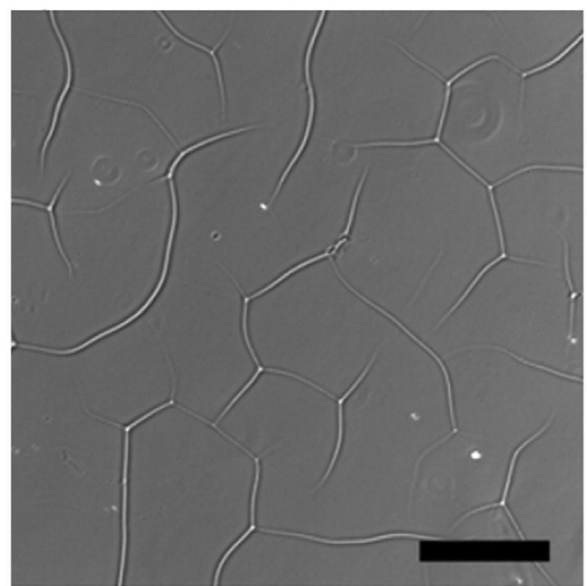

B

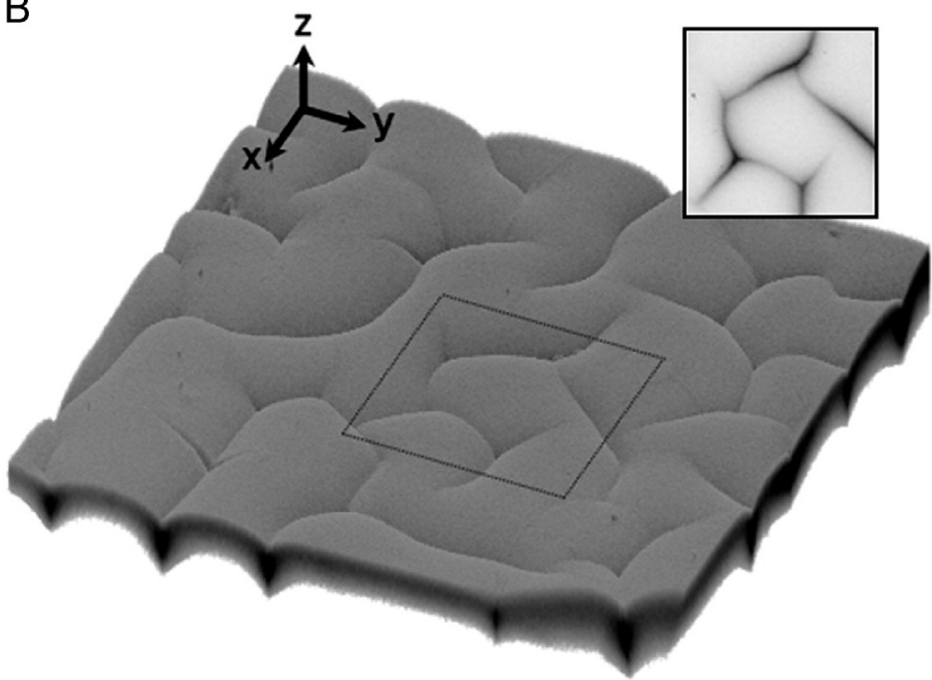

Fig. 1. Surface patterns emerging at the free surface of a swelling acrylamide gel in a Petri dish (top, from Tanaka et al., 1987). Phase-contrast optical image (A, scale bar $200 \mu \mathrm{m}$ ) and confocal fluorescence image (B, inset: slice $32 \mu \mathrm{m}$ below the free surface) of the creased surface of a swelling polyacrylamide gel (bottom, from Saha et al., 2010). 
fully nonlinear instability, they cannot emerge below Biot's threshold unless the system's symmetry is broken (Hohlfeld and Mahadevan, 2011). Furthermore, pattern formation has been mainly studied in the case of uniaxial strain, where creasing has been proved to be a subcritical instability (Cao and Hutchinson, 2012), while only few studies considered morphological transitions in two-dimensional configurations (Kim et al., 2011). The aim of this work is to perform a weakly nonlinear analysis of the wrinkling instability of a soft layer under equi-biaxial compression. The article is organized as follows. The elastic growth model is presented in Section 2, and the nonlinear incremental equation applied over its base homogeneous solution is derived in Section 3. The theory of incremental elastic deformations superimposed on finite strains is used in Section 4 to perform a linear stability analysis. A multiple-scale perturbation method is formulated in Section 5 in order to perform a weakly nonlinear stability analysis in Section 6. The normal form of the elastic bifurcation is derived in Section 7. Finally, the discussion of the results of this study and few conclusive remarks are collected in the last section.

\section{The elastic growth model and its base solution}

In a Cartesian coordinate system with unit base vectors $\mathbf{e}_{i}$, with $i=(x, y, z)$, let me consider a soft elastic layer attached at the surface $Z=0$, and having a vertical thickness $H$ much smaller than the horizontal lengths $L_{x}, L_{y}$. Indicating with $\mathbf{X}=\mathbf{X}(X, Y, Z)$ and $\mathbf{x}=\mathbf{x}(x, y, z)$ its material and spatial position vectors, respectively, the kinematics is described by the geometrical deformation tensor $\mathbf{F}=\partial \mathbf{X} / \partial \mathbf{X}$. The elastic layer can undergo a isotropic volume variation (e.g. swelling for a polymeric gel, or growth for a living material), and its grown natural state is defined by the tensor $\mathbf{F}_{g}=$ gI, where $g$ is the isotropic growth rate and $\mathbf{I}$ is the unit tensor. Such a volumetric growth is modeled by a multiplicative decomposition of $\mathbf{F}$, as follows:

$$
\mathbf{F}=\mathbf{F}_{e} \mathbf{F}_{g}
$$

where $\mathbf{F}_{e}$ is the elastic deformation tensor (Rodriguez et al., 1994). Whenever $\mathbf{F}_{g}$ is not compatible with the spatial constraints, the elastic deformation restores the overall compatibility of the layer's deformation, whilst residual stresses arise.

From a constitutive viewpoint, the body is modeled as an isotropic neo-Hookean incompressible material with a surface energy at the free boundary $Z=H$, so that its total strain energy $W$ reads

$$
W=\frac{\mu}{2} \iiint(\operatorname{tr} \mathbf{b}-3) d X d Y d Z+\gamma \iint_{Z=H}\left(\left|\mathbf{x}_{, X} \times \mathbf{X}_{, Y}\right|\right) d X d Y,
$$

where $\mu$ is the shear modulus, $\mathbf{b}_{e}=\mathbf{F}_{e} \mathbf{F}_{e}^{T}$ is the elastic left Cauchy-Green deformation tensor (having the same trace of the right Cauchy-Green tensor $\mathbf{C}_{e}=\mathbf{F}_{e}^{T} \mathbf{F}_{e}$ ), and $\gamma$ is the surface tension coefficient. The presence of a surface tension in Eq. (2) implies the occurrence of a normal stress at the free surface (i.e. Young-Laplace's law).

The base solution for a growing layer, whose fields are indicated with superscript (0) in the following, corresponds to a homogeneous elastic deformation $\mathbf{F}_{e}^{(0)}=\operatorname{diag}\left(\lambda_{x}, \lambda_{y}, \lambda_{z}\right)$ whose principal stretches read $\lambda_{x}=\lambda_{y}=1 / g$ and $\lambda_{z}=g^{2}$, restoring the compatibility with the constraint of zero displacement at the layer's bottom $Z=0$. Using the constitutive assumptions in Eq. (2), the residual Cauchy stress $\boldsymbol{\sigma}^{(0)}$ and first Piola-Kirchoff stress tensor $\mathbf{P}^{(0)}$ for the layer read

$$
\boldsymbol{\sigma}^{(0)}=\mu \mathbf{b}_{e}^{(0)}-p_{0} \mathbf{I}, \quad \mathbf{P}^{(0)}=\mu \mathbf{F}_{e}^{(0)^{T}}-p_{0} \mathbf{F}_{e}^{(0)}-1
$$

where $p_{0}$ is a Lagrange multiplier ensuring the constraint of incompressibility, i.e. det $\mathbf{F}_{e}=1$, which can be fixed through the stress-free boundary conditions at $Z=H$, as $p_{0}=\lambda_{z}^{2} \mu$. Therefore the layer is subjected to a equi-biaxial stress with $P_{x x}^{(0)}=P_{y y}^{(0)}=\mu\left(g^{-1}-g^{5}\right)$, which is compressive if $g>1$, i.e. if the layer is increasing its volume.

In the following section, the nonlinear governing equations for the incremental problem will be derived.

\section{Nonlinear incremental equations}

In order to investigate the stability of such a homogeneous deformation, the theory of incremental deformations superposed on finite strains will be used (Ogden, 1997). Let me now consider a perturbation with a nonlinear displacement field $\mathbf{u}=\mathbf{u}\left(\mathbf{x}^{(0)}\right)$, so that $\boldsymbol{\Gamma}=\nabla \mathbf{u}=$ grad $\mathbf{u}$ is the displacement gradient with respect to the basis elastic state. Under this assumption, the perturbed elastic deformation gradient reads

$$
\mathbf{F}_{e}=\mathbf{F}_{e}^{(0)}+\delta \mathbf{F}_{e}^{(0)}=\mathbf{F}_{e}^{(0)}+\boldsymbol{\Gamma} \mathbf{F}_{e}^{(0)}
$$

where $\delta \mathbf{F}_{e}^{(0)}=\mathbf{\Gamma} \mathbf{F}_{e}^{(0)}$ is the increment of the base elastic deformation gradient $\mathbf{F}_{e}^{(0)}$. The incremental equations of motion can be written in the spatial form as

$$
\nabla \cdot \mathbf{S}_{0}=\rho \partial_{t t} \mathbf{u}
$$

where $\mathbf{S}_{0}$ is the push-forward of the first Piola-Kirchhoff stress tensor. Making a series expansion in the vicinity of the base elastic state, so that $\mathbf{S}_{0}=\mathbf{S}_{0}^{(0)}+\delta \mathbf{S}_{0}$, the fully nonlinear expression of the incremental stress reads

$$
\delta \mathbf{S}_{0}=\mathbf{L}: \mathbf{\Gamma}+p \mathbf{I}-(p+\delta p) \mathbf{F}_{e}^{(0)} \mathbf{F}_{e}^{-1}
$$

where $\mathbf{L}$ is the forth-order tensor of the instantaneous moduli, i.e. the push-forward of the fixed reference elasticity tensor, and $\delta p$ is the nonlinear incremental pressure associated with the perturbation. In the case of a neo-Hookean material, it is easy to check that the components of $\mathbf{L}$ are simply $L_{j i k l}=\mu b_{j k} \delta_{i l}=\lambda_{j}^{2} \delta_{j k} \delta_{i l}$ in a coordinate system aligned with the directions 
of the principal stretches, whilst higher order instantaneous moduli vanish. Accordingly, using Eq. (4) in Eq. (6) the incremental stress can be rewritten as

$$
\delta \mathbf{S}_{0}=\mu(\widetilde{\nabla} \mathbf{u})^{T}-(p+\delta p) \sum_{m=1}^{\infty}(-1)^{m} \boldsymbol{\Gamma}^{m}-\delta p \mathbf{I}
$$

where I defined the operator $\widetilde{\nabla}=\left[\lambda_{x}^{2} \partial_{x}, \lambda_{y}^{2} \partial_{y}, \lambda_{z}^{2} \partial_{z}\right]^{T}$, and the series development of $(\mathbf{I}+\boldsymbol{\Gamma})^{-1}$ has been used.

Similarly, the incompressibility constraint can be written as

$$
\operatorname{det}(\mathbf{I}+\boldsymbol{\Gamma})=\operatorname{det}\left(\sum^{\infty}(-1)^{m} \boldsymbol{\Gamma}_{m}^{m}\right)_{0}^{-1}=1
$$

Finally, using Eq. (6) in Eq. (5), the governing incremental equations

read

$$
\mu \nabla \cdot(\widetilde{\nabla} \mathbf{u})^{T}-\nabla(\delta p) \sum_{m=0}^{\infty}(-1)^{m} \boldsymbol{\Gamma}^{m}=\rho \partial_{t t} \mathbf{u}
$$

where I used the identity $\nabla \cdot\left(\mathbf{F}_{e}^{(0)} \mathbf{F}_{e}^{-1}\right)=0 .^{1}$

\section{Linear stability analysis for a growing gel layer}

Let me now apply an infinitesimal perturbation using the theory of incremental deformations. Hence let $\mathbf{u}^{(1)}(x, y, z)$ be the infinitesimal incremental displacement field, the linear incremental incompressibility condition imposes that

$$
\nabla \cdot \mathbf{u}^{(1)}=0
$$

From Eq. (6), the constitutive equation for the linear components of the incremental stress $\delta \mathbf{S}^{(1)}$ veads

$$
\delta \mathbf{S}_{0}^{(1)}=\mu\left(\widetilde{\nabla} \mathbf{u}^{(1)}\right)^{T}+p_{0} \nabla \mathbf{u}^{(1)}-\delta p^{(1)} \mathbf{I}
$$

where $\delta p^{(1)}$ is the linear increment in the Lagrange multiplier. In the absence of body forces, I can therefore write the equations of motion as

$$
\nabla \cdot\left(\delta \mathbf{S}_{0}^{(1)}\right)=\rho \partial_{t t} \mathbf{u}^{(1)}
$$

Using Eq. (11), the incremental equations of motion in Eq. (12) take the following simplified form:

$$
-\delta p_{, x}^{(1)}+\mu \lambda_{i}^{2} u_{x, i i}^{(1)}=\rho u_{x, t t}^{(1)}, \quad-\delta p_{, y}^{(1)}+\mu \lambda_{i}^{2} u_{y, i i}^{(1)}=\rho u_{y, t t}^{(1)}, \quad-\delta p_{, z}^{(1)}+\mu \lambda_{i}^{2} u_{z, i i}^{(1)}=\rho u_{z, t t}^{(1)},
$$

where comma denotes partial derivative and Einstein's summation rule on repeated indices is assumed. Differentiating these incremental equations of motion with respect to $x, y$, and $z$, respectively, and using the incremental incompressibility condition in Eq. (10), I find that

$$
\nabla^{2}\left(\delta p^{(1)}\right)=0
$$

implying that the incremental Lagrange multiplier is a Laplacian field (Flavin, 1963).

Now, I consider that the bottom $z=0$ of the layer is fixed (clamped condition), and incremental boundary conditions read

$$
u_{i}^{(1)}(x, y, 0)=0 \text {, }
$$

while the top surface $z=\left(\lambda_{z} / \lambda_{x}\right) H=g^{3} H=h$ remains free of incremental traction, being

$$
\begin{aligned}
& u_{x, z}^{(1)}+u_{z, x}^{(1)}=0, \\
& u_{y, z}^{(1)}+u_{z, y}^{(1)}=0, \\
& -\lambda_{z}^{2} \delta p^{(1)}+2 \mu \lambda_{z}^{2} u_{z, z}^{(1)}-\gamma \lambda_{x}^{2}\left(u_{z, x x}^{(1)}+u_{z, y y}^{(1)}\right)=0 .
\end{aligned}
$$

Let me search for solutions to Eqs. (10), (13), and (14) in the following form:

$$
\left\{u_{x}^{(1)}, u_{y}^{(1)}, u_{z}^{(1)}, \delta p^{(1)}\right\}=\left\{U_{x}^{(1)}(z), U_{y}^{(1)}(z), \imath U_{z}^{(1)}(z), \imath P^{(1)}(z)\right\}\left(e^{\imath k(\cos \theta x+\sin \theta y-v t)}+c . c .\right),
$$

corresponding to the occurrence of a surface wave with wavenumber $k$ and velocity $v$, forming an angle $\theta$ with the $x$-axis, 1 is the imaginary unit and c.c. indicates the complex conjugate. A solution which automatically satisfies the incompressibility condition in Eq. (10) is given by

$$
U_{x}^{(1)}=-\frac{\Psi^{\prime}(z)}{k} \cos \theta, \quad U_{y}^{(1)}=-\frac{\Psi^{\prime}(z)}{k} \sin \theta, \quad U_{z}^{(1)}=\Psi(z)
$$

where the prime denotes differentiation. Accordingly, the equations of motion in Eqs. (13) and (14) read

$$
\mu \lambda_{z}^{2} \Psi^{\prime \prime \prime \prime}-k^{2}\left(\mu\left(\lambda_{x}^{2}+\lambda_{z}^{2}\right)-\rho v^{2}\right) \Psi^{\prime \prime}+k^{4}\left(\mu \lambda_{x}^{2}-\rho v^{2}\right) \Psi=0
$$

\footnotetext{
${ }^{1}$ In fact, considering that $\mathbf{F}_{e}^{(0)}$ is a homogeneous tensor, one has $\operatorname{div}\left(\mathbf{F}_{e}^{(0)} \mathbf{F}_{e}^{-1}\right)=\operatorname{Div} \mathbf{F}_{e}^{-1}=0$.
} 


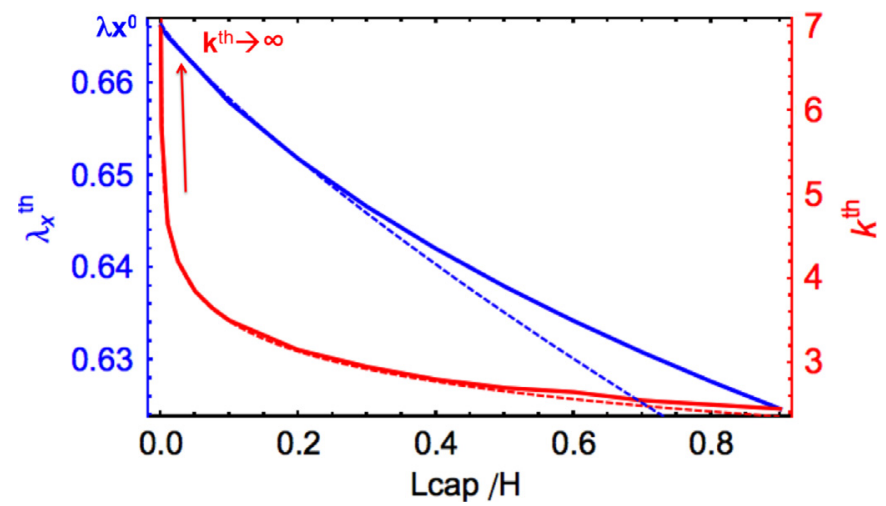

Fig. 2. Dispersion curves showing the critical equi-biaxial compression $\lambda_{x}^{\text {th }}=1 / \mathrm{g}^{\text {th }}$ and the wavenumber $k$ th versus the dimensionless parameter $L_{c a p} / H$. The dashed lines represent the series expansions given by Eqs. (25) and (26).

$$
P_{z}^{(1)}=\frac{\mu \lambda_{z}^{2}}{k^{2}} \Psi^{\prime \prime \prime}-\left(\mu \lambda_{x}^{2}-\rho v^{2}\right) \Psi^{\prime}
$$

An expression for $\Psi=\Psi(z)$ satisfying the boundary conditions in Eq. (15) is given by

$$
\Psi(z)=-\cosh (q k z)+\cosh (k z)+B(q \sinh (k z)-\sinh (q k z))
$$

where $B=\left(2 \cosh (k h)-\left(1+q^{2}\right) \cosh (q k h)\right) /\left(-2 q \sinh (k h)+\left(1+q^{2}\right) \sinh (q k h)\right)$ is imposed by the first of Eq. (16), whilst the value of $q$ is fixed by Eq. (19), being

$$
\left(\lambda_{x}^{2}-\lambda_{z}^{2} q^{2}\right) \mu-\rho v^{2}=0
$$

Considering the last boundary conditions in Eq. (16), the dispersion relation for the linear wave reads

$$
\begin{gathered}
4 \lambda_{z}^{2}\left(q+q^{3}\right)+\cosh (q k h)\left(-\lambda_{z}^{2} q\left(5+2 q^{2}+q^{4}\right) \cosh (k h)-L_{c a p} \lambda_{x}^{2} k q\left(-1+q^{2}\right) \sinh (k h)\right) \\
+\left(L_{c a p} \lambda_{x}^{2} k\left(-1+q^{2}\right) \cosh (k h)+\lambda_{z}^{2}\left(1+6 q^{2}+q^{4}\right) \sinh (k h)\right) \sinh (q k h)=0
\end{gathered}
$$

where $L_{c a p}=\gamma / \mu$ is the capillary length. Accordingly, the elastic problem is governed by the dimensionless parameter $L_{c a p} / H$, which defines the ratio between the capillary and the bulk elastic energies of the body.

\section{Neutrally stable mode and near-critical waves}

The condition of neutral stability of the layer is given setting $v=0$ in Eq. (22), whose solution is $\lambda_{x}=q \lambda_{z}=q / \lambda_{x}^{2}$. If one considers the limit $L_{c a p}=0$, the classical Biot instability occurs for $k \rightarrow \infty$ at the threshold value for $\lambda_{x}=q^{1 / 3}=1 / g$ given by the real root of $\left(-1+3 \lambda_{x}^{3}+\lambda_{x}^{6}+\lambda_{x}^{9}\right)=0$, being

$$
\lambda_{x}^{0}=\left(\frac{-1-\frac{42^{2 / 3}}{(13+3 \sqrt{33})^{1 / 3}}+(2(13+3 \sqrt{33}))^{1 / 3}}{3}\right)^{1 / 3} \simeq 0.6661
$$

which corresponds to a growth threshold given by $g^{0}=1 / \lambda_{x}^{0} \simeq 1.5011$. This is consistent with Biot's prediction for a generalized plain strain condition given by $\lambda_{z}^{0} / \lambda_{x}^{0}=\left(\lambda_{x}^{0}\right)^{-3} \simeq 3.4$ (Hong et al., 2009). Nevertheless, the capillary length regularizes such a singular behavior allowing to fix the wavelength at the onset of the instability (Ben Amar and Ciarletta, 2010), as depicted in Fig. 2.

In particular, an analytical solution can be derived by series expansion within the limit $L_{c a p} / H \ll 1$. Under this assumption, the critical instability thresholds $\lambda_{x}^{\text {th }}$ and $g^{\text {th }}$ read

$$
\lambda_{x}^{\text {th }}=\lambda_{x}^{0}-0.0110345 \frac{L_{\text {cap }}}{H} \log \left[\frac{109.088 H}{L_{\text {cap }}}\right] ; \quad g^{\text {th }}=g^{0}+0.0110345\left(g^{0}\right)^{2} \frac{L_{\text {cap }}}{H} \log \left[\frac{109.088 H}{L_{\text {cap }}}\right]
$$

whilst a logarithmic correction for the wavenumber arises

$$
k^{\text {th }}=\frac{1}{2 H} \log \left[\frac{1.20373}{\lambda_{x}^{0}-\lambda_{x}^{\text {th }}}\right] \simeq \frac{1}{2 H} \log \left[\frac{109.088 H}{L_{\text {cap }}}\right]
$$


A near-critical state is defined taking a small deviation of the threshold value for the growth, such that $\left(g-g^{\text {th }}\right) / g^{\text {th }} \ll 1$. Beyond the threshold, the small velocity of the near-critical wave is given from Eq. (22) as follows:

$$
v=\frac{\sqrt{6 \mu}}{g^{\text {th }} \sqrt{\rho}} \sqrt{\frac{g-g^{t h}}{g^{t h}}}
$$

Therefore, a multiple-scale analysis can be performed for the near-critical mode, considering a characteristic length $L_{c}=1 / k^{\text {th }}$ and a characteristic time $t_{c}=g^{0} \sqrt{\rho} / k^{\text {th }} \sqrt{6 \mu}$. Taking $\epsilon=\sqrt{\left(g-g^{t h}\right) / g^{t h}}$ as the order parameter of the bifurcation, the following scaling applies:

$$
\mathbf{u}=\sum_{\alpha=1}^{\infty} \epsilon^{\alpha} \mathbf{u}^{(\alpha)}(\mathbf{x}, t, \tau) ; \quad \delta p=\sum_{\alpha=1}^{\infty} \epsilon^{\alpha} \delta p^{(\alpha)}(\mathbf{x}, t, \tau)
$$

with $\tau=\epsilon \cdot\left(t / t_{c}\right)$ being a slow time scale which can be treated as an independent variable (Bender and Orszag, 1999). Accordingly, the deformation in the near critical state is defined by

$$
\lambda_{x}=\left(g^{t h}\right)^{-1}\left(1-\epsilon^{2}\right) ; \quad \lambda_{z}=\left(g^{t h}\right)^{2}\left(1+2 \epsilon^{2}\right) ; \quad p=\mu\left(g^{t h}\right)^{4}\left(1+4 \epsilon^{2}\right)
$$

In the following, a weakly nonlinear analysis will be performed using the scaling argument and the series developments in Eqs. (28) and (29).

\section{Weakly nonlinear stability analysis}

The multiple-scale analysis in Eq. (28) is applied in the following for investigating the weakly nonlinear emergence of surface patterns. A superposition of $(n+1)$ linear modes is considered, such that $\mathbf{u}^{(1)}=\sum_{j=0}^{n} \mathbf{u}_{j}^{(1)}$, with

$$
\begin{aligned}
& u_{x}^{(1)}=-\frac{\Psi^{\prime}(z)}{k} \sum_{j=0}^{n} \cos \theta_{j}\left(A_{n}(\tau) e^{\imath k\left(x \cos \theta_{j}+y \sin \theta_{j}\right)}+\text { c.c. }\right) \\
& u_{y}^{(1)}=-\frac{\Psi^{\prime}(z)}{k} \sum_{j=0}^{n} \sin \theta_{j}\left(A_{n}(\tau) e^{\imath k\left(x \cos \theta_{j}+y \sin \theta_{j}\right)}+\text { c.c. }\right) \\
& u_{z}^{(1)}=\Psi(z) \sum_{j=0}^{n}\left(\imath A_{n}(\tau) e^{l k\left(x \cos \theta_{j}+y \sin \theta_{j}\right)}+\text { c.c. }\right) \\
& \delta p^{(1)}=P^{(1)}(z) \sum_{j=0}^{n}\left(\imath A_{n}(\tau) e^{l k\left(x \cos \theta_{j}+y \sin \theta_{j}\right)}+\text { c.c. }\right)
\end{aligned}
$$

where $\theta_{j}=(j \pi / n)$ for $n=(1,2)$ and $\theta_{j}=0$ for $n=0, P^{(1)}(z)$ and $\Psi(z)$ are given by Eqs. (20) and (21) at the neutrally stable mode, and $A_{n}(\tau)$ are the wave amplitudes that must be fixed by nonlinear effects. In particular, the occurrence of a monochromatic mode (cylinders, $n=0$ ), of two coupled orthogonal modes (squares, $n=1$ ) and three interacting waves (hexagons, $n=2$ ) will be examined. The linear modes having different direction of propagation $\theta$ appear concurrently because of the rotational invariance, so that the proposed expression is a combination of interacting modes with a phase shift given by $\theta_{n}$. The amplitudes $A_{n}(\tau)$ are assumed to be independent on the wave direction in order to respect the original symmetry of the elastic problem. In the following paragraph, the second-order solutions of these modes will be derived.

\subsection{Second-order solutions of the incremental equations}

At the second order in $\epsilon$, the incompressibility condition in Eq. (8) takes the following form:

$$
\nabla \cdot \mathbf{u}^{(2)}-\frac{1}{2} \operatorname{tr}\left[\left(\boldsymbol{\Gamma}^{(1)}\right)^{2}\right]=0
$$

where $\boldsymbol{\Gamma}^{(1)}=\nabla \mathbf{u}^{(1)}$, and it has been made use of Eq. (10).

The equations of motion in Eq. (9) read

$$
\mu \nabla \cdot\left(\widetilde{\nabla} \mathbf{u}^{(2)}\right)^{T}-\nabla(\delta p)^{(2)}+\mu\left(\nabla \cdot\left(\widetilde{\nabla} \mathbf{u}^{(1)}\right)^{T}\right) \cdot \nabla \mathbf{u}^{(1)}=0
$$

where Eq. (12) has been used for eliminating the dependence on $\delta p^{(1)}$. Similarly, cross-differentiation of Eq. (35) allows to make the equations independent on $\delta p^{(2)}$, as follows:

$$
\begin{aligned}
& \left(\nabla \cdot\left(\widetilde{\nabla} u_{x}^{(2)}\right)^{T}\right)_{, y}-\left(\nabla \cdot\left(\widetilde{\nabla} u_{y}^{(2)}\right)^{T}\right)_{, x}+\left(\left(\nabla \cdot\left(\widetilde{\nabla} \mathbf{u}^{(1)}\right)^{T}\right) \cdot \mathbf{u}_{, x}^{(1)}\right)_{, y}-\left(\left(\nabla \cdot\left(\widetilde{\nabla} \mathbf{u}^{(1)}\right)^{T}\right) \cdot \mathbf{u}_{, y}^{(1)}\right)_{, x}=0 \\
& \left(\nabla \cdot\left(\widetilde{\nabla} u_{x}^{(2)}\right)^{T}\right)_{, z}-\left(\nabla \cdot\left(\widetilde{\nabla} u_{z}^{(2)}\right)^{T}\right)_{, x}+\left(\left(\nabla \cdot\left(\widetilde{\nabla} \mathbf{u}^{(1)}\right)^{T}\right) \cdot \mathbf{u}_{, x}^{(1)}\right)_{, z}-\left(\left(\nabla \cdot\left(\widetilde{\nabla} \mathbf{u}^{(1)}\right)^{T}\right) \cdot \mathbf{u}_{, z}^{(1)}\right)_{, x}=0 \\
& \left(\nabla \cdot\left(\widetilde{\nabla} u_{z}^{(2)}\right)^{T}\right)_{, y}-\left(\nabla \cdot\left(\widetilde{\nabla} u_{y}^{(2)}\right)^{T}\right)_{, z}+\left(\left(\nabla \cdot\left(\widetilde{\nabla} \mathbf{u}^{(1)}\right)^{T}\right) \cdot \mathbf{u}_{, z}^{(1)}\right)_{, y}-\left(\left(\nabla \cdot\left(\widetilde{\nabla} \mathbf{u}^{(1)}\right)^{T}\right) \cdot \mathbf{u}_{, y}^{(1)}\right)_{, z}=0
\end{aligned}
$$


The boundary conditions at the layer's substrate read

$$
\mathbf{u}^{(2)}=\mathbf{0} \quad \text { at } z=0
$$

while the stress-free condition at the free surface imposes

$$
\mathbf{e}_{z} \delta \mathbf{S}_{0}^{(2)}=\mathbf{f}_{\text {cap }} \quad \text { at } z=h
$$

with

$$
\begin{aligned}
\mathbf{f}_{c a p}= & {\left[\lambda_{x}^{2} \gamma u_{z, x}^{(1)}\left(u_{z, y y}^{(1)}+u_{z, x x}^{(1)}\right), \lambda_{x}^{2} \gamma u_{z, y}^{(1)}\left(u_{z, y y}^{(1)}+u_{z, x x}^{(1)}\right), \lambda_{x}^{2} \gamma\left(-u_{z, y y}^{(2)}+2\left(u_{x, y}^{(1)}+u_{y, x}^{(1)}\right) u_{z, x y}^{(1)}\right.\right.} \\
& +u_{z, x}^{(1)}\left(u_{x, y y}^{(1)}+u_{x, x x}^{(1)}\right)+u_{z, y}^{(1)}\left(u_{y, y y}^{(1)}+u_{y, x x}^{(1)}\right) \\
& \left.\left.+\left(u_{y, y}^{(1)}-u_{x, x}^{(1)}\right)\left(u_{z, y y}^{(1)}-u_{z, x x}^{(1)}\right)-u_{z, x x}^{(2)}\right)\right]^{T}
\end{aligned}
$$

and the second-order stress component is given by Eq. (7), being

$$
\delta \mathbf{S}_{0}^{(2)}=\mu\left(\widetilde{\nabla} \mathbf{u}^{(2)}\right)^{T}-p_{0}\left(\left(\boldsymbol{\Gamma}^{(1)}\right)^{2}-\boldsymbol{\Gamma}^{(2)}\right)+\delta p^{(1)} \boldsymbol{\Gamma}^{(1)}-\delta p^{(2)} \mathbf{I}
$$

For the sake of simplicity, the boundary conditions involving $\left(\delta S_{0}^{(2)}\right)_{z z}$ can be rewritten by differentiating Eq. (40) and using Eq. (35) for eliminating the dependence on $\delta p^{(2)}$. Furthermore, although the capillary force allows at the linear order to regularize the singular behavior of Biot's problem, thus fixing the wavelength of the neutrally stable mode, $\mathbf{f}_{\text {cap }}$ can be neglected when solving the second-order problem. In fact, since singularities are avoided at the linear order, it can be easily checked that the higher order term $\left.\mathbf{f}_{c a p} / \mu=\alpha \gamma / \mu\right)$ imposes a very small correction in the second-order solution and it will not be considered in the following.

If $n$ is the subscript indicating the linear mode in Eqs. (30)-(33), the second order solution has $n^{2}+2(n+1)$ nonlinear interactions of the linear modes, so the solution will be given by imposing Eqs. (34) and (35) at each of these interaction orders. In the following, the second order solution is given for the three linear solutions under consideration.

\subsubsection{Second-order solution for the cylinders}

The second-order solution for the cylindrical mode, i.e. setting $n=0$ in Eqs. (30)-(33), is derived in the following. In particular, the second-order displacement fields contain quadratic nonlinear interactions with the linear solution, so it can be expressed as follows:

$$
u_{i}^{(2)}=U_{i}^{(2)}(z)\left|A_{0}(\tau)\right|^{2}+\left(V_{i}^{(2)}(z) A_{0}^{2}(\tau) e^{2 l k x}+\text { c.c. }\right)
$$

with $i=(x, y, z)$. After lengthy manipulations, the displacement fields are derived from the solutions of Eqs. (34) and (35) with boundary conditions Eqs. (39) and (40) at each interacting mode. In particular, the following expressions have been derived:

$$
\begin{aligned}
& U_{x}^{(2)^{\prime \prime}}=0 \quad \text { with } U_{x}^{(2)}(0)=U_{x}^{(2) \prime}(h)=U_{x}^{(2)^{\prime \prime}}(h)=0 \\
& U_{y}^{(2)^{\prime \prime}}=0 \quad \text { with } U_{y}^{(2)}(0)=U_{y}^{(2) \prime}(h)=U_{y}^{(2)^{\prime \prime}}(h)=0 \\
& U_{z}^{(2) \prime}=2\left(U_{z}^{(1)} U_{z}^{(1))^{\prime}}\right)^{\prime} \quad \text { with } U_{z}^{(2)}(0)=0
\end{aligned}
$$

whose solution is simply given by

$$
U_{x}^{(2)}=U_{y}^{(2)}=0 ; \quad U_{z}^{(2)}=2 U_{z}^{(1)} U_{z}^{(1)}
$$

Finally, the remaining displacement fields read

$$
\begin{aligned}
& V_{y}^{(2)}=0 ; \quad V_{x}^{(2)}=l \frac{U_{z}^{(1) \prime 2}-U_{z}^{(1)} U_{z}^{(1)^{\prime \prime}}+V_{z}^{(2) \prime}}{2 k} \\
& \lambda_{z}^{2} V_{z}^{(2)^{\prime \prime \prime}}-4 k^{2}\left(\lambda_{x}^{2}+\lambda_{z}^{2}\right) V_{z}^{(2)^{\prime \prime}}+16 k^{4} \lambda_{x}^{2} V_{z}^{(2)}=3 k^{2}\left(\lambda_{z}^{2}-\lambda_{x}^{2}\right)\left(U_{z}^{(1)} U_{z}^{(1)^{\prime \prime}}-U_{z}^{(1)^{\prime}} U_{z}^{(1)^{\prime \prime}}\right)
\end{aligned}
$$

having the following boundary conditions:

$$
\begin{aligned}
& V_{z}^{(2)}(0)=V_{z}^{(2) \prime}(0)=0 \\
& k^{2}\left(4 \lambda_{z}^{2} V_{z}^{(2)}(h)-\left(\lambda_{x}^{2}+7 \lambda_{z}^{2}\right) U_{z}^{(1)}(h) U_{z}^{(1) \prime}(h)\right)+\lambda_{z}^{2} V_{z}^{(2)^{\prime \prime}}(h)=0 \\
& -4\left(\lambda_{x}^{2}-2 \lambda_{z}^{2}\right) k^{2} U_{z}^{(1) \prime 2}(h)-4\left(\lambda_{x}^{2}+2 \lambda_{z}^{2}\right) k^{2} V_{z}^{(2) \prime}(h)+\lambda_{z}^{2} V_{z}^{(2) " \prime}(h)=0
\end{aligned}
$$

where the linear boundary conditions in Eq. (16) have been used for simplifying. The solution of Eq. (49) is given by

$$
V_{z}^{(2)}=e^{-\left(2 \lambda_{x} k z\right) / \lambda_{z}} v_{1}+e^{\left(2 \lambda_{x} k z\right) / \lambda_{z}} v_{2}+e^{-2 k z} v_{3}+e^{2 k z} v_{4}+b_{1} U_{z}^{(1) \prime} U_{z}^{(1)^{\prime \prime}}+b_{2} U_{z}^{(1)} U_{z}^{(1)^{\prime \prime}}
$$


with

$$
b_{2}=\frac{9\left(\lambda_{x}^{2} \lambda_{z}^{2}-\lambda_{z}^{4}\right)}{k^{2}\left(9 \lambda_{x}^{4}-82 \lambda_{x}^{2} \lambda_{z}^{2}+9 \lambda_{z}^{4}\right)} \simeq \frac{-4.314}{k^{2}} ; \quad b_{1}=-\frac{7 b_{2}}{3} \simeq \frac{10.067}{k^{2}}
$$

where $v_{1}, \ldots, v_{4}$ are constant parameters which are fixed by the boundary conditions in Eqs. (50)-(52). Their analytical expression is very cumbersome and it is not enclosed in this article for the sake of simplicity.

In conclusion, Eqs. (43), (47), and (53) represent the second order displacement field of the perturbed solution for the cylindrical mode. It is interesting to notice that the second order solution is independent on the direction of the monochromatic mode, as shown in Appendix A.

\subsubsection{Second-order solution for the squares}

The second-order solution for the squares, i.e. setting $n=1$ in Eqs. (30)-(33), can be expressed in the following form:

$$
\begin{aligned}
u_{i}^{(2)}= & U_{i}^{(2)}(z)\left|A_{1}(\tau)\right|^{2}+\left(V_{1 i}^{(2)}(z) A_{1}^{2}(\tau) e^{2 l k x}+V_{2 i}^{(2)}(z) A_{1}^{2}(\tau) e^{2 l k y}\right. \\
& \left.+W_{1 i}^{(2)}(z) A_{1}^{2}(\tau) e^{i k(x+y)}+W_{2 i}^{(2)}(z)\left|A_{1}(\tau)\right|^{2} e^{i k(x-y)}+\text { c.c. }\right)
\end{aligned}
$$

with $i=(x, y, z)$. The self-interacting modes do not depend on the orientation of the linear wave (see Appendix A), therefore it is straightforward to show that

$$
V_{2 x}^{(2)}=V_{1 y}^{(2)}=0 ; \quad V_{1 z}^{(2)}=V_{2 z}^{(2)}=V_{z}^{(2)} ; \quad V_{1 x}^{(2)}=V_{2 y}^{(2)}=\imath \frac{U_{z}^{(1), 2}-U_{z}^{(1)} U_{z}^{(1)^{\prime \prime}}+V_{1 z}^{(2) \prime}}{2 k}
$$

together with

$$
U_{x}^{(2)}=U_{y}^{(2)}=0 ; \quad U_{z}^{(2)}=4 U_{z}^{(1)} U_{z}^{(1) \prime}
$$

After lengthy manipulations on Eqs. (34) and (35), the other second order displacement fields for the squares can be expressed as

$$
W_{2 z}^{(2)}=-W_{1 z}^{(2)} ; \quad W_{1 x}^{(2)}=W_{1 y}^{(2)}=W_{2 y}^{(2)}=-W_{2 x}^{(2)}=l \frac{U^{(1)^{\prime} 2}-W_{2 z}^{(2) \prime}}{2 k}
$$

where $W_{2 z}^{(2)}$ is the solution of the following differential equation:

$$
\begin{aligned}
\lambda_{z}^{2} W_{2 z}^{(2)^{\prime \prime \prime}} & -2 k^{2}\left(\lambda_{x}^{2}+\lambda_{z}^{2}\right) W_{2 z}^{(2)^{\prime \prime}}+4 k^{4} \lambda_{x}^{2} W_{2 z}^{(2)} \\
& =-2 k^{2}\left(\lambda_{x}^{2}-2 \lambda_{z}^{2}\right) U_{z}^{(1)} U_{z}^{(1)^{\prime \prime}}+6 \lambda_{z}^{2} U_{z}^{(1)^{\prime \prime}} U_{z}^{(1)^{\prime \prime}}-2 U_{z}^{(1)}\left(\lambda_{x}^{2} k^{4} U_{z}^{(1)^{\prime}}+\lambda_{z}^{2} k^{2} U_{z}^{(1)^{\prime \prime \prime}}\right)
\end{aligned}
$$

According to Eq. (59), the solution for $W_{2 z}^{(2)}$ takes the following expression:

$$
\begin{aligned}
W_{2 z}^{(2)}= & e^{-\left(\sqrt{2} \lambda_{x} k z\right) / \lambda_{z}} W_{1}+e^{\left(\sqrt{2} \lambda_{x} k z\right) / \lambda_{z}} W_{2}+e^{-\sqrt{2} k z} W_{3}+e^{\sqrt{2} k z} W_{4} \\
& +d_{1} U_{z}^{(1) \prime} U_{z}^{(1)^{\prime \prime}}+d_{2} U_{z}^{(1)^{\prime \prime}} U_{z}^{(1)^{\prime \prime}}+d_{3} U_{z}^{(1)^{\prime}} U_{z}^{(1)}+d_{4} U_{z}^{(1)} U_{z}^{(1)^{\prime \prime}}
\end{aligned}
$$

with

$$
\begin{aligned}
& d_{1}=-\frac{2 \lambda_{z}^{2}\left(\lambda_{x}^{4}-\lambda_{x}^{2} \lambda_{z}^{2}+2 \lambda_{z}^{4}\right)}{\left(-2 \lambda_{x}^{2}+\lambda_{z}^{2}\right)\left(\lambda_{x}^{4}-6 \lambda_{x}^{2} \lambda_{z}^{2}+\lambda_{z}^{4}\right) k^{2}} \simeq \frac{9.62787}{k^{2}} ; \quad d_{2}=\frac{\lambda_{z}^{4}\left(\lambda_{x}^{2}+3 \lambda_{z}^{2}\right)}{\left(-2 \lambda_{x}^{2}+\lambda_{z}^{2}\right)\left(\lambda_{x}^{4}-6 \lambda_{x}^{2} \lambda_{z}^{2}+\lambda_{z}^{4}\right) k^{4}} \simeq \frac{7.73982}{k^{4}} ; \\
& d_{3}=\frac{2 \lambda_{x}^{6}-13 \lambda_{x}^{4} \lambda_{z}^{2}-\lambda_{x}^{2} \lambda_{z}^{4}}{2 \lambda_{x}^{6}-13 \lambda_{x}^{4} \lambda_{z}^{2}+8 \lambda_{x}^{2} \lambda_{z}^{4}-\lambda_{z}^{6}} \simeq 0.464527 ; \quad d_{4}=\frac{2 \lambda_{z}^{2}}{\left(-2 \lambda_{x}^{2}+\lambda_{z}^{2}\right) k^{2}} \simeq \frac{2.42353}{k^{2}}
\end{aligned}
$$

The constant parameters $w_{1}, \ldots, w_{4}$ can be derived by solving the four following boundary conditions from Eqs. (39) and (40) being

$$
\begin{aligned}
& W_{2 z}^{(2)}(0)=W_{2 z}^{(2) \prime}(0)=0 \\
& 2 k^{2}\left(W_{2 z}^{(2)}(h)+2 U_{z}^{(1)}(h) U_{z}^{(1) \prime}(h)\right)+\left(W_{2 z}^{(2)^{\prime \prime}}\right)(h)=0 \\
& 2 k^{2}\left(-\lambda_{x}^{2} W_{2 z}^{(2) \prime}(h)-\lambda_{z}^{2}\left(k^{2} U_{z}^{(1) 2}(h)+2 W_{2 z}^{(2) \prime}(h)+4 U_{z}^{(1) \prime 2}(h)\right)\right. \\
& \left.\quad+\left(\lambda_{x}^{2}+\lambda_{z}^{2}\right) U_{z}^{(1)}(h) U_{z}^{(1)^{\prime \prime}}(h)\right)+\lambda_{z}^{2} W_{2 z}^{(2)^{\prime \prime \prime}}(h)=0
\end{aligned}
$$

Finally, Eqs. (55)-(58) and (60) represent the second order displacement field of the perturbed solution for the squares. 
6.1.3. Second-order solution for the hexagons

The second-order solution for the hexagons, i.e. setting $n=2$ in Eqs. (30)-(33), must take the following form:

$$
\begin{aligned}
& u_{i}^{(2)}=U_{i}^{(2)}(z)\left|A_{2}(\tau)\right|^{2} \\
& +\left(V_{2 i}^{(2)}(z) A_{2}^{2}(\tau) e^{2 \imath k x}+V_{2 i}^{(2)}(z) A_{2}^{2}(\tau) e^{i k(x+\sqrt{3} y)}+V_{3 i}^{(2)}(z) A_{2}^{2}(\tau) e^{-i k(x-\sqrt{3} y)}\right. \\
& +W_{1 i}^{(2)}(z) A_{2}^{2}(\tau) e^{(1 / 2) k(3 x+\sqrt{3} y)}+W_{2 i}^{(2)}(z) A_{2}^{2}(\tau) e^{i k \sqrt{3} y}+W_{3 i}^{(2)}(z)\left|A_{2}(\tau)\right|^{2} e^{-(1 / 2) l k(3 x-\sqrt{3} y)} \\
& \left.+Y_{1 i}^{(2)}(z)\left|A_{2}(\tau)\right|^{2} e^{(1 / 2) l k(x-\sqrt{3} y)}+Y_{2 i}^{(2)}(z)\left|A_{2}(\tau)\right|^{2} e^{i k x}+Y_{3 i}^{(2)}(z) A_{2}^{2}(\tau) e^{(1 / 2) k(x+\sqrt{3} y)}+\text { c.c. }\right)
\end{aligned}
$$

with $i=(x, y, z)$. As for the previous case, the analysis on the self-interacting modes allows to derive the following relations:

$$
\begin{aligned}
& V_{2 x}^{(2)}=-V_{3 x}^{(2)}=\frac{V_{2 y}^{(2)}}{\sqrt{3}}=\frac{V_{3 y}^{(2)}}{\sqrt{3}}=\frac{V_{1 x}^{(2)}}{2}=l \frac{U_{z}^{(1) \prime 2}-U_{z}^{(1)} U_{z}^{(1)^{\prime \prime}}+V_{1 z}^{(2) \prime}}{4 k} ; \\
& V_{1 y}^{(2)}=0 ; \quad V_{1 z}^{(2)}=V_{2 z}^{(2)}=V_{3 z}^{(2)}=V_{z}^{(2)}
\end{aligned}
$$

together with

$$
U_{x}^{(2)}=U_{y}^{(2)}=0 ; \quad U_{z}^{(2)}=6 U_{z}^{(1)} U_{z}^{(1) \prime}
$$

After lengthy manipulations on Eqs. (34) and (35), the solution for the hexagons can be calculated by solving two differential equations of the forth-order in $W_{2 z}^{(2)}$ and $Y_{2 z}^{(2)}$.

The first set of solutions is given by

$$
\begin{aligned}
& W_{3 x}^{(2)}=-\frac{3}{\sqrt{3}} W_{3 y}^{(2)}=W_{1 x}^{(2)}=\frac{3}{\sqrt{3}} W_{1 y}^{(2)}=\frac{\sqrt{3}}{2} W_{2 y}^{(2)}=l \frac{5 U_{z}^{(1) \prime 2}-4 U_{z}^{(1)} U_{z}^{(1)^{\prime \prime}}+4 W_{2 z}^{(2) \prime}}{8 k} ; \\
& W_{2 x}^{(2)}=0 ; \quad W_{1 z}^{(2)}=W_{2 z}^{(2)}=-W_{3 z}^{(2)}
\end{aligned}
$$

where $W_{2 z}^{(2)}$ is the solution of the following differential equation:

$$
\begin{aligned}
& 2 \lambda_{z}^{2} W_{2 z}^{(2)^{\prime \prime \prime}}-6 k^{2}\left(\lambda_{x}^{2}+\lambda_{z}^{2}\right) W_{2 z}^{(2)^{\prime \prime}}+18 k^{4} \lambda_{x}^{2} W_{2 z}^{(2)} \\
& \quad=\left(-7 \lambda_{x}^{2}+8 \lambda_{z}^{2}\right) k^{2} U_{z}^{(1) \prime} U_{z}^{(1)^{\prime \prime}}+4\left(\left(\lambda_{x}^{2}-2 \lambda_{z}^{2}\right) k^{2} U_{z}^{(1)}+\lambda_{z}^{2} U_{z}^{(1)^{\prime \prime}}\right) U_{z}^{(1)^{\prime \prime \prime}}
\end{aligned}
$$

According to Eq. (69), the solution for $W_{2 z}^{(2)}$ takes the following expression:

$$
\begin{aligned}
W_{2 z}^{(2)}= & e^{-\left(\sqrt{3} \lambda_{x} k z\right) / \lambda_{z}} W_{1}+e^{\left(\sqrt{3} \lambda_{x} k z\right) / \lambda_{z}} W_{2}+e^{-\sqrt{3} k z} W_{3}+e^{\sqrt{3} k z} W_{4} \\
& +d_{1} U_{z}^{(1) \prime} U_{z}^{(1)^{\prime \prime}}+d_{2} U_{z}^{(1)^{\prime \prime \prime}} U_{z}^{(1)^{\prime \prime}}+d_{3} U_{z}^{(1) \prime} U_{z}^{(1)}+d_{4} U_{z}^{(1)} U_{z}^{(1)^{\prime \prime \prime}}
\end{aligned}
$$

with

$$
\begin{aligned}
& d_{1}=\frac{3 \lambda_{z}^{2}\left(24 \lambda_{x}^{8}-74 \lambda_{x}^{6} \lambda_{z}^{2}+93 \lambda_{x}^{4} \lambda_{z}^{4}-102 \lambda_{x}^{2} \lambda_{z}^{6}+32 \lambda_{z}^{8}\right)}{2\left(-16 \lambda_{x}^{10}+172 \lambda_{x}^{8} \lambda_{z}^{2}-444 \lambda_{x}^{6} \lambda_{z}^{4}+403 \lambda_{x}^{4} \lambda_{z}^{6}-136 \lambda_{x}^{2} \lambda_{z}^{8}+12 \lambda_{z}^{10}\right) k^{2}} \simeq \frac{12.253}{k^{2}} ; \\
& d_{2}=\frac{3 \lambda_{z}^{4}\left(\lambda_{x}^{2}+2 \lambda_{z}^{2}\right)\left(6 \lambda_{x}^{4}-15 \lambda_{x}^{2} \lambda_{z}^{2}+5 \lambda_{z}^{4}\right)}{\left(16 \lambda_{x}^{10}-172 \lambda_{x}^{8} \lambda_{z}^{2}+444 \lambda_{x}^{6} \lambda_{z}^{4}-403 \lambda_{x}^{4} \lambda_{z}^{6}+136 \lambda_{x}^{2} \lambda_{z}^{8}-12 \lambda_{z}^{10}\right) k^{4}} \simeq-\frac{8.04551}{k^{4}} ; \\
& d_{3}=\frac{-8 \lambda_{x}^{10}+98 \lambda_{x}^{8} \lambda_{z}^{2}-171 \lambda_{x}^{6} \lambda_{z}^{4}+5 \lambda_{x}^{4} \lambda_{z}^{6}+22 \lambda_{x}^{2} \lambda_{z}^{8}}{16 \lambda_{x}^{10}-172 \lambda_{x}^{8} \lambda_{z}^{2}+444 \lambda_{x}^{6} \lambda_{z}^{4}-403 \lambda_{x}^{4} \lambda_{z}^{6}+136 \lambda_{x}^{2} \lambda_{z}^{8}-12 \lambda_{z}^{10}} \simeq-0.637068 \\
& d_{4}=\frac{3 \lambda_{z}^{2}\left(8 \lambda_{x}^{8}-60 \lambda_{x}^{6} \lambda_{z}^{2}+115 \lambda_{x}^{4} \lambda_{z}^{4}-56 \lambda_{x}^{2} \lambda_{z}^{6}+8 \lambda_{z}^{8}\right)}{\left(16 \lambda_{x}^{10}-172 \lambda_{x}^{8} \lambda_{z}^{2}+444 \lambda_{x}^{6} \lambda_{z}^{4}-403 \lambda_{x}^{4} \lambda_{z}^{6}+136 \lambda_{x}^{2} \lambda_{z}^{8}-12 \lambda_{z}^{10}\right) k^{2}} \simeq-\frac{4.07122}{k^{2}}
\end{aligned}
$$

The constant parameters $w_{1}, \ldots, w_{4}$ can be derived by solving the four following boundary conditions from Eqs. (39) and (40) being

$$
\begin{aligned}
& W_{2 z}^{(2)}(0)=W_{2 z}^{(2) \prime}(0)=0 \\
& k^{2}\left(3 \lambda_{z}^{2} W_{2 z}^{(2)}(h)-\left(\lambda_{x}^{2}+8 \lambda_{z}^{2}\right) U_{z}^{(1)}(h) U_{z}^{(1) \prime}(h)\right)+\lambda_{z}^{2} W_{2 z}^{(2)^{\prime \prime}}(h)=0 \\
& 2\left(4 \lambda_{x}^{2}+5 \lambda_{z}^{2}\right) k^{4} U_{z}^{(1) 2}(h)+k^{2}\left(\left(-13 \lambda_{x}^{2}+40 \lambda_{z}^{2}\right) U_{z}^{(1) \prime 2}(h)-12\left(\lambda_{x}^{2}+2 \lambda_{z}^{2}\right) W_{2 z}^{(2) \prime}(h)\right)+4 \lambda_{z}^{2} W_{2 z}^{(2)^{\prime \prime \prime}}(h)=0
\end{aligned}
$$


The last set of remaining solutions is given by

$$
\begin{gathered}
Y_{1 x}^{(2)}=-\frac{Y_{1 y}^{(2)}}{3}=-Y_{3 x}^{(2)}=-\frac{Y_{3 y}^{(2)}}{\sqrt{3}}=\frac{Y_{2 x}^{(2)}}{2}=l \frac{4 Y_{2 z}^{(2) \prime}-\left(5 U_{z}^{(1) / 2}+4 U_{z}^{(1)} U_{z}^{(1)^{\prime \prime}}\right)}{8 k} ; \\
Y_{2 y}^{(2)}=0 ; \quad Y_{1 z}^{(2)}=Y_{2 z}^{(2)}=-Y_{3 z}^{(2)}
\end{gathered}
$$

where $Y_{2 z}^{(2)}$ is the solution of the following differential equation:

$$
2 \lambda_{z}^{2} Y_{2 z}^{(2)^{\prime \prime \prime}}-2 k^{2}\left(\lambda_{x}^{2}+\lambda_{z}^{2}\right) Y_{2 z}^{(2)^{\prime \prime}}+2 k^{4} \lambda_{x}^{2} Y_{2 z}^{(2)}=3\left(-4 \lambda_{x}^{2} k^{4} U_{z}^{(1)} U_{z}^{(1) \prime}+U_{z}^{(1)^{\prime \prime}}\left(\left(\lambda_{x}^{2}+4 \lambda_{z}^{2}\right) k^{2} U_{z}^{(1) \prime}+8 \lambda_{z}^{2} U_{z}^{(1)^{\prime \prime \prime}}\right)\right)
$$

According to Eq. (76), the solution for $Y_{2 z}^{(2)}$ takes the following expression:

$$
\begin{aligned}
Y_{2 z}^{(2)}= & e^{-\left(\lambda_{x} k z\right) / \lambda_{z}} y_{1}+e^{\left(\lambda_{x} k z\right) / \lambda_{z}} y_{2}+e^{-k z} y_{3}+e^{k z} y_{4} \\
& +f_{1} U_{z}^{(1) \prime} U_{z}^{(1)^{\prime \prime}}+f_{2} U_{z}^{(1) \prime} U_{z}^{(1)}+f_{3} U_{z}^{(1)} U_{z}^{(1)^{\prime \prime \prime}}
\end{aligned}
$$

with

$$
\begin{aligned}
& f_{1}=-\frac{3 \lambda_{z}^{2}}{2\left(-4 \lambda_{x}^{2}+\lambda_{z}^{2}\right) k^{2}} \simeq-2.30596 ; \quad f_{2}=\frac{6 \lambda_{x}^{2}}{4 \lambda_{x}^{2}-\lambda_{z}^{2}} \simeq-0.805961 ; \\
& f_{3}=\frac{3 \lambda_{z}^{2}}{\left(-4 \lambda_{x}^{2}+\lambda_{z}^{2}\right) k^{2}} \simeq 4.61192
\end{aligned}
$$

The constant parameters $y_{1}, \ldots, y_{4}$ can be derived by solving the four following boundary conditions from Eqs. (39) and (40) being

$$
\begin{aligned}
& Y_{2 z}^{(2)}(0)=Y_{2 z}^{(2) \prime}(0)=0 \\
& k^{2}\left(\lambda_{z}^{2} Y_{2 z}^{(2)}(h)-\left(\lambda_{x}^{2}-2 \lambda_{z}^{2}\right) U_{z}^{(1) \prime}(h) U_{z}^{(1)}(h)\right)+\lambda_{z}^{2} Y_{2 z}^{(2)^{\prime \prime}}(h)=0 \\
& 18 \lambda_{z}^{2} k^{4} U_{z}^{(1) 2}(h)+k^{2}\left(4\left(\lambda_{x}^{2}+2 \lambda_{z}^{2}\right) Y_{2 z}^{(2) \prime}(h)+\left(13 \lambda_{x}^{2}+40 \lambda_{z}^{2}\right) U_{z}^{(1) \prime 2}(h)\right)-4 \lambda_{z}^{2} Y_{2 z}^{(2)^{\prime \prime \prime}}(h)=0
\end{aligned}
$$

Finally, Eqs. (65)-(68), (70), (75), and (77) represent the second order displacement field of the perturbed solution for the hexagons. Once calculated the second-order solutions for the problem, it is possible to derive the amplitude equations for the elastic instability.

\subsection{Derivation of the amplitude equations}

In the previous section, the second-order solutions of the incremental problem have been derived for the three patterns under consideration. A further series development of the solution would introduce resonant terms in the third-order displacement fields, because of the cubic nonlinear interactions of the linear modes (Fu and Rogerson, 1994). Therefore, a number of amplitude or Ginzburg-Landau equations should be introduced in order to avoid the occurrence of secular terms in the solution of the incremental equations.

Nevertheless, the full derivation of the third-order elastic solution of this problem would be very hard to get and it is not considered in the following. A more elegant method for deriving the amplitude equations, based on energetic considerations (Fu and Ogden, 1999), is presented in the following. In particular, the total elastic energy of the body will be expanded in series of the order parameter $\epsilon$ using the scaling laws in Eqs. (28) and (29). Since the second-order solutions of the incremental problem contain nonlinear quadratic terms of interaction with the linear mode, it is expected that solvability conditions must be imposed to ensure the energy conservation at the fourth order expansion in $\epsilon$.

Let $U$ be the total elastic energy of the body in the perturbed volume $V_{p}$ of the instability, as follows:

$$
\left.U=\int_{V_{p}} \psi_{v}+\rho \frac{\mathbf{u}_{, t}^{2}}{2}\right) d v+\int_{S_{p}} \gamma d s
$$

where $S_{p}$ indicates the free surface in the perturbed configuration and $\psi_{v}$ is the neo-Hookean bulk strain energy density, whose series development around the perturbed solution at $\mathbf{F}_{e}$ is given by

$$
\psi_{v}=\psi_{v 0}+\frac{\mu}{2} \operatorname{tr}\left[\mathbf{b}_{e} \nabla \mathbf{u}\right]+\frac{\mu}{4} \operatorname{tr}\left[(\widetilde{\nabla} \mathbf{u})^{T} \nabla \mathbf{u}\right]=\psi_{v 0}+p \nabla \cdot \mathbf{u}+\operatorname{tr}[\boldsymbol{\sigma} \nabla \mathbf{u}]+\frac{\mu}{4} \operatorname{tr}\left[(\widetilde{\nabla} \mathbf{u})^{T} \nabla \mathbf{u}\right]
$$

where $\psi_{v 0}=\mu\left(\lambda_{z}^{2}+2 \lambda_{x}^{2}-3\right) / 2$. This phenomenon suggests that at a weakly nonlinear regime the doubling of the wavenumber of the instability might occur. 
Recalling from simple differentiation rules and the divergence theorem that

$$
\int \operatorname{tr}[\boldsymbol{\sigma} \nabla \mathbf{u}] d V=-\int_{V}(\nabla \cdot \boldsymbol{\sigma}) \mathbf{u} d V+\int_{S} \mathbf{N} \boldsymbol{\sigma} \mathbf{u} d S=0
$$

where $\mathbf{N}$ is the unit normal vector in the base solution, the total potential energy in Eq. (82) simplifies as:

$$
U=\int_{V}\left(\psi_{v 0}+p \nabla \cdot \mathbf{u}+\frac{\mu}{2} \operatorname{tr}\left[(\widetilde{\nabla} \mathbf{u})^{T} \nabla \mathbf{u}\right]+\epsilon^{2} \rho \frac{\mathbf{u}_{, \tau}^{2}}{2 t_{c}^{2}}\right) d V+\int_{S_{f}} \gamma J_{S} d S
$$

where $S_{f}$ indicates the free surface in the base configuration, and the surface Jacobian is defined as

$J_{S}=\left|(\mathbf{x}+\mathbf{u})_{, x} \wedge(\mathbf{x}+\mathbf{u})_{, y}\right|=J \mathbf{N}(\mathbf{I}+\boldsymbol{\Gamma})^{-1} \mathbf{n}$ by simple manipulations of Nanson's formula, where $\mathbf{n}$ is the perturbed unit normal. relation: Before developing the elastic energy $U$ in Eq. (85) at the leading orders in $\epsilon$, it is useful to introduce the following

$$
\begin{aligned}
\int_{S} \mathbf{N} \delta \mathbf{S}_{0}^{(1)} \mathbf{u}^{(k)} d S & =\int_{V} \operatorname{tr}\left[\left(\mu\left(\widetilde{\nabla} \mathbf{u}^{(1)}\right)^{T}+p \nabla \mathbf{u}^{(1)}-\delta p^{(1)} \mathbf{I}\right) \nabla \mathbf{u}^{(k)}\right] d V \\
& =\int_{S_{f}} \gamma \lambda_{x}^{2}\left(u_{z, x x}^{(1)}+u_{z, y y}^{(1)}\right) \mathbf{e}_{z} \cdot \mathbf{u}^{(k)} d S=O(\gamma)
\end{aligned}
$$

which is derived using Eqs. (11), (12), and (16)), where $\mathbf{u}^{(k)}$ is the generic incremental displacement of order $\epsilon^{k}$. Setting a series development $U=\sum_{k} U^{(k)} \epsilon^{k}$ in Eq. (85) together with Eqs. (28) and (29), the first order term is

$$
U^{(1)}=\int_{V}\left(p_{0} \nabla \cdot \mathbf{u}^{(1)}\right) d V+\int_{S_{f}} \gamma \lambda_{x}^{\text {th }} 2\left(u_{x, x}^{(1)}+u_{y, y}^{(1)}\right) d S=0
$$

where the incompressibility condition Eq. (10) and the periodicity of the perturbation have been used.

The second order term can be simplified using Eq. (10), (34), (86) as follows:

$$
\begin{aligned}
U^{(2)} & =\int_{V}\left(\frac{p_{0}}{2} \operatorname{tr}\left[\nabla \mathbf{u}^{(1)} \nabla \mathbf{u}^{(1)}\right]+\frac{\mu}{2} \operatorname{tr}\left[\left(\widetilde{\nabla} \mathbf{u}^{(1)}\right)^{T} \nabla \mathbf{u}^{(1)}\right]\right) d V+\int_{S_{f}} \gamma J_{S}^{(2)} d S \\
& \left.=\gamma \int_{S_{f}} \frac{\lambda_{x}^{\text {th }} 2}{2}\left(u_{z, x x}^{(1)}+u_{z, y y}^{(1)}\right) u_{z}^{(1)}+J_{S}^{(2)}\right) d S=0
\end{aligned}
$$

where Eqs. (30)-(32) have been used, together with

$$
\left.J_{S}^{(2)}=\lambda_{x}^{\text {th }} 2 \quad u_{y, y}^{(2)}+u_{x, x}^{(2)}+\frac{u_{z, y}^{(1)} 2}{2}+\frac{u_{z, x}^{(1)} 2}{2}+u_{y, y}^{(1)} u_{x, x}^{(1)}-u_{x, y}^{(1)} u_{y, x}^{(1)}\right)
$$

Developing the incompressibility condition Eq. (8) at the third order in $\epsilon$, being

$$
\nabla \cdot \mathbf{u}^{(3)}-\operatorname{tr}\left[\nabla \mathbf{u}^{(1)} \nabla \mathbf{u}^{(2)}\right]+J^{(3)}=0
$$

with $J^{(3)}=\operatorname{det}\left[\nabla \mathbf{u}^{(1)}\right]=\operatorname{det} \Gamma^{(1)}$, i.e. being the third order component of $J=\operatorname{det} \boldsymbol{\Gamma}=\sum_{m} J^{(m)} \epsilon^{m}$, it is straightforward to show that

$$
U^{(3)}=\int_{V}\left(p_{0} \nabla \cdot \mathbf{u}^{(3)}+\frac{\mu}{2} \operatorname{tr}\left[\left(\widetilde{\nabla} \mathbf{u}^{(1)}\right)^{T} \nabla \mathbf{u}^{(2)}+\left(\widetilde{\nabla} \mathbf{u}^{(2)}\right)^{T} \nabla \mathbf{u}^{(1)}\right]\right) d V+\int_{S_{f}} \gamma J_{S}^{(3)} d S=0
$$

which is identically satisfied for cylinders and squares thanks to the periodicity of the integrands. For hexagons, instead, vanishing of Eq. (91) is fulfilled by considering that the amplitude $A_{2}(\tau)$ is a real number, i.e. by enforcing the inversion symmetry $\mathbf{u}^{(1)} \rightarrow-\mathbf{u}^{(1)}$, or $A_{2}(\tau) \rightarrow-A_{2}(\tau)$. In particular, the inversion symmetry $\mathbf{u}^{(1)} \rightarrow-\mathbf{u}^{(1)}$ imposes the absence of quadratic nonlinearities for hexagonal patterns, which would otherwise occur if the amplitudes $A_{2}(\tau)$ were assumed to be dependent on the wave direction.

Similarly, at the fourth-order in $\epsilon$, the elastic energy reads

$$
\begin{aligned}
U^{(4)}= & \int_{V}\left(p_{0} \nabla \cdot \mathbf{u}^{(4)}+2 p_{0} \operatorname{tr}\left[\nabla \mathbf{u}^{(1)} \nabla \mathbf{u}^{(1)}\right]+\frac{\mu}{2} \operatorname{tr}\left[\left(\widetilde{\nabla} \mathbf{u}^{(2)}\right)^{T} \nabla \mathbf{u}^{(2)}\right]+\mu \operatorname{tr}\left[\left(\widetilde{\nabla} \mathbf{u}^{(3)}\right)^{T} \nabla \mathbf{u}^{(1)}\right]\right. \\
& \left.+\frac{\mu}{2} \operatorname{tr}\left[\left(\bar{\nabla} \mathbf{u}^{(1)}\right)^{T} \nabla \mathbf{u}^{(1)}\right]+\rho \frac{\left(\mathbf{u}_{\tau}^{(1)}\right)^{2}}{2 t_{c}^{2}}\right) d V+\int_{S_{f}} \gamma J_{S}^{(4)} d S
\end{aligned}
$$

where $\bar{\nabla}=\left[-2 \lambda_{x}^{\text {th }} 2 \partial_{x x},-2 \lambda_{x}^{\text {th }} 2 \partial_{y y}, 4 \lambda_{z}^{\text {th }} 2 \partial_{z z}\right]^{T}$ and Eqs. (28) and (29) have been used. It is useful considering the following expansion for the incompressibility condition in Eq. (8):

$$
\nabla \cdot \mathbf{u}^{(4)}-\operatorname{tr}\left[\nabla \mathbf{u}^{(1)} \nabla \mathbf{u}^{(3)}\right]-\frac{1}{2} \operatorname{tr}\left[\nabla \mathbf{u}^{(2)} \nabla \mathbf{u}^{(2)}\right]+\frac{1}{2}\left(\nabla \cdot \mathbf{u}^{(2)}\right)^{2}+J^{(4)}=0
$$

with:

$$
\begin{aligned}
J^{(4)}= & u_{z, z}^{(2)} u_{y, y}^{(1)} u_{x, x}^{(1)}-u_{y, z}^{(2)} u_{z, y}^{(1)} u_{x, x}^{(1)}+u_{z, z}^{(1)} u_{y, y}^{(2)} u_{x, x}^{(1)}-u_{y, z}^{(1)} u_{z, y}^{(2)} u_{x, x}^{(1)}-u_{z, z}^{(2)} u_{x, y}^{(1)} u_{y, x}^{(1)}+u_{x, z}^{(2)} u_{z, y}^{(1)} u_{y, x}^{(1)} \\
& -u_{z, z}^{(1)} u_{x, y}^{(2)} u_{y, x}^{(1)}+u_{x, z}^{(1)} u_{z, y}^{(2)} u_{y, x}^{(1)}+u_{y, z}^{(2)} u_{x, y}^{(1)} u_{z, x}^{(1)}-u_{x, z}^{(2)} u_{y, y}^{(1)} u_{z, x}^{(1)}+u_{y, z}^{(1)} u_{x, y}^{(2)} u_{z, x}^{(1)}-u_{x, z}^{(1)} u_{y, y}^{(2)} u_{z, x}^{(1)}
\end{aligned}
$$




$$
+u_{z, z}^{(1)} u_{y, y}^{(1)} u_{x, x}^{(2)}-u_{y, z}^{(1)} u_{z, y}^{(1)} u_{x, x}^{(2)}-u_{z, z}^{(1)} u_{x, y}^{(1)} u_{y, x}^{(2)}+u_{x, z}^{(1)} u_{z, y}^{(1)} u_{y, x}^{(2)}+u_{y, z}^{(1)} u_{x, y}^{(1)} u_{z, x}^{(2)}-u_{x, z}^{(1)} u_{y, y}^{(1)} u_{z, X}^{(2)}
$$

Substituting Eqs. (34), (86), and (93), it is possible to simplify the elastic energy in Eq. (92) as follows:

$$
\begin{aligned}
U^{(4)}= & \int_{V}\left(p_{0}\left(\frac{1}{2} \operatorname{tr}\left[\nabla \mathbf{u}^{(2)} \nabla \mathbf{u}^{(2)}\right]-\frac{1}{2}\left(\nabla \cdot \mathbf{u}^{(2)}\right)^{2}-J^{(4)}+2 \operatorname{tr}\left[\nabla \mathbf{u}^{(1)} \nabla \mathbf{u}^{(1)}\right]\right)\right. \\
& +\delta p^{(1)}\left(\operatorname{tr}\left[\nabla \mathbf{u}^{(1)} \nabla \mathbf{u}^{(2)}\right]-J^{(3)}\right)+\frac{\mu}{2} \operatorname{tr}\left[\left(\widetilde{\nabla} \mathbf{u}^{(2)}\right)^{T} \nabla \mathbf{u}^{(2)}\right] \\
& \left.+\frac{\mu}{2} \operatorname{tr}\left[\left(\bar{\nabla} \mathbf{u}^{(1)}\right)^{T} \nabla \mathbf{u}^{(1)}\right]+\rho \frac{\left(\mathbf{u}_{, \tau}^{(1)}\right)^{2}}{2 t_{c}^{2}}\right) d V \\
& +\gamma \int_{S_{f}}\left(\lambda_{x}^{t h} 2\left(u_{z, x x}^{(1)}+u_{z, y y}^{(1)}\right) u_{z}^{(3)}+J_{S}^{(4)}\right) d S
\end{aligned}
$$

In summary, it is found from Eqs. (87), (88), (91), and (95) that the energy deviation from the base state is of order $\epsilon^{4}$. Such an incremental elastic energy $U^{(4)}$ not only should be bounded in the perturbed configuration but it must also be conserved during the incremental motion for a perfectly elastic body. Therefore, the amplitude equations can be derived by imposing:

$$
d U^{(4)} / d \tau=0
$$

whose expression only requires the analytical solutions for $\mathbf{u}^{(1)}$ and $\mathbf{u}^{(2)}$ for each mode under considerations. In the following section, the amplitude equations for the cylindrical, the square and the hexagonal patterns are derived.

\section{Amplitude equations for cylinders, squares and hexagons}

\subsection{Symmetry and normal form of the bifurcation}

The method for deriving the amplitude equations from energy considerations has been introduced in the previous section. Before calculating their detailed expression for the modes considered in this work, it is useful to investigate the symmetry restrictions on the normal form of the bifurcation.

Considering the periodicity of the base perturbations, the incremental energy per unit of periodic surface can be written as

$$
\begin{aligned}
\delta U= & \frac{k_{x} k_{y}}{4 \pi^{2}} \int_{x=0}^{2 \pi / k_{x}} \int_{y=0}^{2 \pi / k_{y}} \int_{z=0}^{h}\left(p_{0}\left(\frac{1}{2} \operatorname{tr}\left[\nabla \mathbf{u}^{(2)} \nabla \mathbf{u}^{(2)}\right]-\frac{1}{2}\left(\nabla \cdot \mathbf{u}^{(2)}\right)^{2}-J^{(4)}+2 \operatorname{tr}\left[\nabla \mathbf{u}^{(1)} \nabla \mathbf{u}^{(1)}\right]\right)\right. \\
& \left.+\delta p^{(1)}\left(\operatorname{tr}\left[\nabla \mathbf{u}^{(1)} \nabla \mathbf{u}^{(2)}\right]-J^{(3)}\right)+\frac{\mu}{2}\left(\operatorname{tr}\left[\left(\widetilde{\nabla} \mathbf{u}^{(2)}\right)^{T} \nabla \mathbf{u}^{(2)}\right]+\operatorname{tr}\left[\left(\bar{\nabla} \mathbf{u}^{(1)}\right)^{T} \nabla \mathbf{u}^{(1)}\right]\right)+\rho \frac{\left(\mathbf{u}_{, \tau}^{(1)}\right)^{2}}{2 t_{c}^{2}}\right) d V
\end{aligned}
$$

where $k_{x}, k_{y}$ are the wavenumbers of the pattern in the $x, y$ directions, respectively. The conservation of the differential elastic energy in Eq. (96) over a time shift $U(t)=U\left(t+t_{0}\right)$ is equivalent to the transformation of the amplitudes $A_{n}(\tau) \rightarrow$ $A_{n}(\tau) e^{1 \epsilon t_{0} / t_{c}}$. Furthermore, it is easy to show that an inversion invariance also applies from the symmetry of the governing equations. In fact, the differential elastic energy in Eq. (97) is invariant by a transformation $A_{n}(\tau) \rightarrow-A_{n}(\tau)$, or equivalently $\mathbf{u}^{(1)} \rightarrow-\mathbf{u}^{(1)}$, which also implies $\delta p^{(1)} \rightarrow-\delta p^{(1)}, J^{(3)} \rightarrow-J^{(3)}, J^{(4)} \rightarrow J^{(4)}$ and $\mathbf{u}^{(2)} \rightarrow \mathbf{u}^{(2)}$. From both these invariance requirements, the resulting differential energy $\delta U_{n}$ must be in the form

$$
\delta U_{n}=\beta_{n}\left|\frac{\partial A_{n}(\tau)}{\partial \tau}\right|^{2}+\alpha_{1 n}\left|A_{n}(\tau)\right|^{2}+\alpha_{2 n}\left|A_{n}(\tau)\right|^{4}
$$

where $\beta_{n}, \alpha_{1 n}, \alpha_{2 n}$ must be real constants. The normal form of the amplitude equations can be derived by substituting Eq. (98) into Eq. (96), reading

$$
\frac{\partial^{2} A_{n}(\tau)}{\partial \tau^{2}}=r A_{n}(\tau)+\nu_{n}\left|A_{n}(\tau)\right|^{2} A_{n}(\tau)
$$

where $r=-\alpha_{1 n} / \beta_{n}$ is the linear stability coefficient, $\nu_{n}=-2 \alpha_{2 n} / \beta_{n}$ is the nonlinear term, and the conjugate equation is also imposed. The normal form of the elastic bifurcation in Eq. (99) is imposed by the symmetries of the elastic problem: in fact, the governing equations possess the discrete symmetries of parity $\mathbf{x} \rightarrow-\mathbf{x}$ (i.e. $A_{n} \rightarrow A_{n}$ ), and the combined symmetry of time translation $t \rightarrow\left(t+t_{0}\right)$ (i.e. $\left.A_{n} \rightarrow A_{n} e^{1 v t_{0}}\right)$ and of inversion (i.e. $\left.A_{n} \rightarrow-A_{n}\right)$. In particular, the inversion symmetry imposes the absence of quadratic nonlinearities in Eq. (99), avoiding transcritical bifurcations for hexagonal patterns (Golubitsky et al., 1984).

The amplitude equations in Eq. (99) correspond to a pitchfork bifurcation, which will be supercritical (resp. subcritical) if $r / \nu_{n}<0$ (resp. $r / \nu_{n}>0$ ).

The term $r$ only depends on the linear incremental solution regardless of the superposition of different linear modes (Newell and Whitehead, 1969). From Eq. (97), it is straightforward to show that for a single mode (i.e. setting $n=0$ in 
Eqs. (30)-(33)) one has

$$
\begin{aligned}
& \beta_{0}=\frac{\rho}{t_{c}^{2}} \int_{z=0}^{H}\left(\Psi^{2}+\Psi^{\prime 2}\right) d z>0 \\
& \alpha_{10} / \mu=8 \lambda_{z}^{t h} 2 \Psi(h) \Psi^{\prime}(h)+2 \int_{z=0}^{h}\left(-\lambda_{x}^{t h} 2\left(\Psi^{2}+\Psi^{\prime 2}\right)+2 \lambda_{z}^{t h} 2\left(\Psi^{\prime 2}+\Psi^{\prime \prime 2}\right)\right) d z
\end{aligned}
$$

For multiple modes the resulting coefficients are the sum of the ones for each superposing mode, i.e. $\alpha_{1 n}=(n+1) \alpha_{10}$ and $\beta_{n}=(n+1) \beta_{0}$, so that $r=-\alpha_{10} / \beta_{0}$. On the contrary, the nonlinear term $\nu_{n}$ will depend on the orientation of the superposing linear modes, and will be calculated in the following for the three modes under consideration in this work.

\subsection{Derivation of the nonlinear terms in the amplitude equations}

Imposing $L_{c}=1 / k^{\text {th }}=1$ as the unit length and using Eqs. (43)-(48), after lengthy manipulations on Eq. (97) the nonlinear term $\alpha_{20}$ for the cylindrical mode can be expressed as

$$
\begin{aligned}
& \alpha_{20}=\frac{1}{4}\left(16 \lambda_{x}^{t h^{2}} V_{z}^{(2)^{2}}+8\left(\lambda_{x}^{t h^{2}}+\lambda_{z}^{t h^{2}}\right) \Psi^{\prime 4}+4\left(\lambda_{x}^{t h^{2}}+3 \lambda_{z}^{t h^{2}}\right) V_{z}^{(2)^{\prime 2}}\right. \\
& +4\left(\lambda_{x}^{\text {th }}{ }^{2}+2 \lambda_{z}^{\text {th }}{ }^{2}\right) \Psi^{2} \Psi^{\prime \prime 2}+\Psi^{\prime 2}\left(8 \lambda_{z}^{\text {th }} V_{z}^{(2)^{\prime}}+\Psi^{\prime \prime}\left(2\left(\lambda_{x}^{\text {th }}{ }^{2}+8 \lambda_{z}^{\text {th }}{ }^{2}\right) \Psi+\lambda_{z}^{\text {th }} \Psi^{\prime \prime}\right)\right) \\
& +\lambda_{z}^{t h^{2}} V_{z}^{(2)^{\prime \prime 2}}-4 \lambda_{z}^{t h^{2}} \Psi^{\prime 3} \Psi^{\prime \prime \prime}-\lambda_{z}^{t{ }^{2}} \Psi^{2} \Psi^{\prime \prime 2}-8\left(\lambda_{x}^{t h^{2}}+\lambda_{z}^{t h^{2}}\right) \Psi V_{z}^{(2)^{\prime}} \Psi^{\prime \prime} \\
& +2 \Psi^{\prime}\left(\lambda_{x}^{t h^{2}} \Psi^{2} \Psi^{\prime \prime \prime}+\lambda_{z}^{t h^{2}}\left(\Psi^{\prime \prime} V_{z}^{(2)^{\prime \prime}}+4 V_{z}^{(2)} \Psi^{\prime \prime \prime}\right)-\Psi\left(\lambda_{x}^{t h^{2}} V_{z}^{(2)^{\prime \prime}}+6 \lambda_{z}^{\text {th }}{ }^{2} \Psi^{\prime \prime} \Psi^{\prime \prime \prime}\right)\right) \\
& \left.+8 V_{z}^{(2)}\left(\left(-\lambda_{x}^{\text {th }}{ }^{2}+\lambda_{z}^{t h^{2}}\right) \Psi^{\prime} \Psi^{\prime \prime}+\lambda_{z}^{\text {th }}{ }^{2}\left(V_{z}^{(2)^{\prime \prime}}+\left(-\Psi+\Psi^{\prime \prime}\right) \Psi^{\prime \prime \prime}\right)\right)\right)
\end{aligned}
$$

where the terms $\Psi$ and $V_{z}^{(2)}$ are given by Eqs. (21) and (53), respectively.

Similarly, using Eqs. (55)-(58), the nonlinear term $\alpha_{21}$ for the squares (i.e. setting $n=1$ in Eqs. (30)-(33)) is given by

$$
\begin{aligned}
& \alpha_{21}=\frac{1}{2}\left(2 \Psi ^ { \prime \prime \prime } \left(-2 \lambda_{x}^{t^{2}{ }^{2}} \Psi^{2} \Psi^{\prime}+\lambda_{z}^{\text {th }^{2}} \Psi\left(4 W_{2 z}^{\prime \prime}+9 \Psi^{\prime} \Psi^{\prime \prime}-3 V_{1 z}^{\prime \prime}-4 V_{1 z}\right)\right.\right. \\
& \left.+4 \lambda_{z}^{\text {th }}{ }^{2}\left(\Psi^{\prime}\left(3 W_{2 z}^{\prime}+2 \Psi^{\prime 2}-2 V_{1 z}^{\prime}\right)+2\left(W_{2 z}-V_{1 z}\right) \Psi^{\prime \prime}\right)\right) \\
& -16\left(\lambda_{x}^{\text {th }}{ }^{2}+\lambda_{z}^{\text {th }}{ }^{2}\right) W_{2 z} \Psi^{\prime} \Psi^{\prime \prime}+8\left(2 \lambda_{x}^{\text {th }}{ }^{2}+\lambda_{z}^{\text {th }}{ }^{2}\right) V_{1 z} \Psi^{\prime} \Psi^{\prime \prime}-8 \lambda_{x}^{\text {th }}{ }^{2} \Psi W_{2 z}^{\prime \prime} \Psi^{\prime}-32 \lambda_{x}^{\text {th }}{ }^{2} W_{2 z}^{\prime} \Psi^{\prime 2} \\
& +4 \lambda_{x}^{t h^{2}} W_{2 z}^{\prime 2}+8 \lambda_{x}^{t h^{2}} W_{2 z}^{2}-8 \lambda_{x}^{t h^{2}} \Psi \Psi^{\prime \prime} V_{1 z}^{\prime}+4 \lambda_{x}^{t h^{2}} \Psi^{2} \Psi^{\prime \prime 2}+4 \lambda_{x}^{t h^{2}} \Psi \Psi^{\prime} V_{1 z}^{\prime \prime}+24 \lambda_{x}^{\text {th }}{ }^{2} \Psi^{\prime 2} V_{1 z}^{\prime} \\
& -8 \lambda_{x}^{t h^{2}} \Psi^{\prime 4}-28 \lambda_{x}^{t h^{2}} \Psi \Psi^{\prime 2} \Psi^{\prime \prime}+4 \lambda_{x}^{t h^{2}} V_{1 z}^{\prime}{ }^{2}+16 \lambda_{x}^{t h^{2}} V_{1 z}^{2}-8 \lambda_{z}^{t h^{2}} \Psi^{\prime} \Psi^{\prime \prime}+2 \lambda_{z}^{t h^{2}} W_{2 z}^{\prime \prime}+8 \lambda_{z}^{t h^{2}} W_{2 z} W_{2 z}^{\prime \prime} \\
& -8 \lambda_{z}^{t h^{2}} W_{2 z}^{\prime} \Psi^{\prime 2}+12 \lambda_{z}^{t h^{2}} W_{2 z}^{\prime 2}+5 \lambda_{z}^{t h^{2}} \Psi^{2} \Psi^{\prime \prime 2}-8 \lambda_{z}^{t h^{2}} \Psi \Psi^{\prime \prime} V_{1 z}^{\prime}+16 \lambda_{z}^{t h^{2}} \Psi^{2} \Psi^{\prime \prime 2}+8 \lambda_{z}^{t h^{2}} \Psi^{\prime 2} V_{1 z}^{\prime} \\
& +16 \lambda_{z}^{t h^{2}} \Psi^{\prime 4}+2 \lambda_{z}^{t t^{2}} \Psi^{\prime} \Psi^{\prime \prime} V_{1 z}^{\prime \prime}+9 \lambda_{z}^{t h^{2}} \Psi^{\prime 2} \Psi^{\prime \prime 2}+32 \lambda_{z}^{t t^{2}} \Psi \Psi^{\prime 2} \Psi^{\prime \prime}+\lambda_{z}^{t h^{2}} V_{1 z}^{\prime \prime}+8 \lambda_{z}^{t t^{2}} V_{1 z} V_{1 z}^{\prime \prime} \\
& \left.+12 \lambda_{z}^{t^{2}} V_{1 z}^{\prime 2}\right)
\end{aligned}
$$

where the terms $\Psi, V_{2 z}^{(2)}$ and $W_{2 z}^{(2)}$ are given by Eqs. (21), (56), and (60), respectively.

Finally, using Eqs. (65)-(68), the nonlinear term $\alpha_{22}$ for the hexagons (i.e. setting $n=2$ in Eqs. (30)-(33)) reads

$$
\begin{aligned}
& \alpha_{22}=\frac{1}{8}\left(24 \lambda_{x}^{\text {th }} Y_{2 z}^{(2)^{2}}+96 \lambda_{x}^{t^{2}} V_{2 z}^{(2)^{2}}+72 \lambda_{x}^{t^{2}} W_{2 z}^{(2)^{2}}+24 \lambda_{x}^{t^{2}{ }^{2}} Y_{2 z}^{(2)^{\prime 2}}+72 \lambda_{z}^{\text {th }} Y_{2 z}^{(2)^{\prime 2}}\right. \\
& -180 \lambda_{x}^{\text {th }}{ }^{2} Y_{2 z}^{(2)} \Psi^{\prime 2}-168 \lambda_{z}^{\text {th }}{ }^{2} Y_{2 z}^{(2)^{\prime}} \Psi^{\prime 2}+123 \lambda_{x}^{\text {th }} \Psi^{\prime 4}+264 \lambda_{z}^{\text {th }}{ }^{2} \Psi^{\prime 4}+144 \lambda_{x}^{\text {th }}{ }^{\prime 2} \Psi^{\prime 2} V_{2 z}^{(2)^{\prime}} \\
& +48 \lambda_{z}^{t h^{2}} \Psi^{\prime 2} V_{2 z}^{(2)^{\prime}}+24 \lambda_{x}^{t h^{2}} V_{2 z}^{(2)^{\prime 2}}+72 \lambda_{z}^{t^{2}{ }^{2}} V_{2 z}^{(2)^{\prime 2}}+228 \lambda_{x}^{t h^{2}} \Psi^{\prime 2} W_{2 z}^{(2)^{\prime}}+120 \lambda_{z}^{\text {th }}{ }^{2} \Psi^{\prime 2} W_{2 z}^{(2)^{\prime}} \\
& +24 \lambda_{x}^{t^{2}} W_{2 z}^{(2)^{\prime 2}}+72 \lambda_{z}^{t^{2}} W_{2 z}^{(2)^{\prime 2}}+48 \lambda_{z}^{t^{2}{ }^{2}} Y_{2 z}^{(2)} Y_{2 z}^{(2)^{\prime \prime}}-48 \lambda_{x}^{t h^{2}} \Psi \Psi^{\prime} Y_{2 z}^{(2) "}-72 \lambda_{z}^{\text {th }}{ }^{2} \Psi \Psi^{\prime} Y_{2 z}^{(2)^{\prime \prime}} \\
& +24 \lambda_{z}^{t h^{2}} Y_{2 z}^{(2)}{ }^{\prime \prime 2}-48 \lambda_{x}^{t h^{2}} \Psi Y_{2 z}^{(2)} \Psi^{\prime \prime}-120 \lambda_{z}^{t h^{2}} \Psi Y_{2 z}^{(2)} \Psi^{\prime \prime}-48\left(\lambda_{x}^{t^{2}}+5 \lambda_{z}^{t h^{2}}\right) Y_{2 z}^{(2)} \Psi^{\prime} \Psi^{\prime \prime} \\
& +48\left(2 \lambda_{x}^{\text {th }}{ }^{2}+\lambda_{z}^{\text {th }}{ }^{2}\right) V_{2 z}^{(2)} \Psi^{\prime} \Psi^{\prime \prime}+144 \lambda_{x}^{\text {th }}{ }^{2} W_{2 z}^{(2)} \Psi^{\prime} \Psi^{\prime \prime}+144 \lambda_{z}^{\text {th }}{ }^{2} W_{2 z}^{(2)} \Psi^{\prime} \Psi^{\prime \prime}-24 \lambda_{x}^{\text {th }} \Psi^{2} \Psi \Psi^{\prime 2} \Psi^{\prime \prime} \\
& +744 \lambda_{z}^{t h^{2}} \Psi \Psi^{\prime 2} \Psi^{\prime \prime}-48 \lambda_{x}^{t h^{2}} \Psi V_{2 z}^{(2)} \Psi^{\prime \prime}-48 \lambda_{z}^{t^{2}} \Psi V_{2 z}^{(2)^{\prime}} \Psi^{\prime \prime}-48 \lambda_{x}^{\text {th }}{ }^{2} \Psi W_{2 z}^{(2)} \Psi^{\prime \prime} \\
& -24 \lambda_{z}^{t h^{2}} \Psi W_{2 z}^{(2)^{\prime}} \Psi^{\prime \prime}-168 \lambda_{z}^{t h^{2}} \Psi^{\prime} Y_{2 z}^{(2){ }^{\prime \prime}} \Psi^{\prime \prime}+72 \lambda_{x}^{t h^{2}} \Psi^{2} \Psi^{\prime \prime 2}+192 \lambda_{z}^{t h^{2}} \Psi^{2} \Psi^{\prime \prime 2}+318 \lambda_{z}^{t h^{2}} \Psi^{\prime 2} \Psi^{\prime \prime 2}
\end{aligned}
$$


Table 1

Coefficients of the Ginzburg-Landau equations.

\begin{tabular}{lrrr}
\hline$L_{\text {cap }} / H$ & $r$ & $\nu_{0}$ & $\nu_{1}$ \\
\hline 0.001 & 1.001 & $8.54 \times 10^{15}$ & $3.18 \times 10^{15}$ \\
0.025 & 0.999 & $-6.73 \times 10^{9}$ & $-8.72 \times 10^{7}$ \\
\hline
\end{tabular}
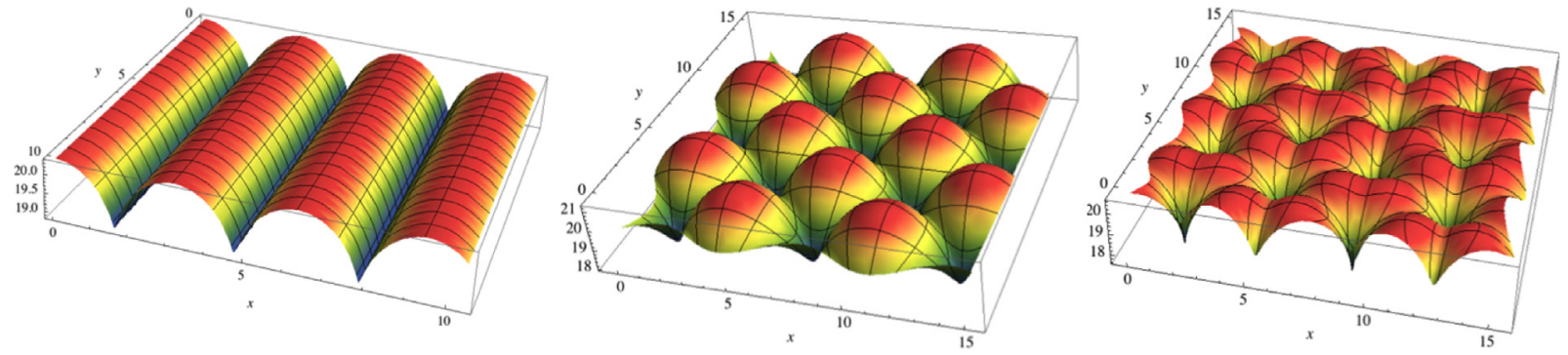

Fig. 3. Weakly nonlinear solutions of the morphology of the layer's top surface for cylinders (left: $n=0, A_{0}=0.08$ ), squares (center: $n=1, A_{1}=0.0025$ ), and hexagons (right: $n=2, A_{2}=0.025$ ) with $\epsilon=\sqrt{\left(g-g^{\text {th }}\right) / g^{\text {th }}}=0.001, L_{\text {cap }} / H=0.001, k^{\text {th }}=1$.

$$
\begin{aligned}
& +48 \lambda_{z}^{t h^{2}} V_{2 z}^{(2)} V_{2 z}^{(2)^{\prime \prime}}+24 \lambda_{x}^{t h^{2}} \Psi \Psi^{\prime} V_{2 z}^{(2)^{\prime \prime}}+12 \lambda_{z}^{t h^{2}} \Psi^{\prime} \Psi^{\prime \prime} V_{2 z}^{(2)^{\prime \prime}}+6 \lambda_{z}^{t h^{2}} V_{2 z}^{(2)^{\prime \prime}}+48 \lambda_{z}^{t h^{2}} W_{2 z}^{(2)} W_{2 z}^{(2)^{\prime \prime}} \\
& +48 \lambda_{x}^{t h^{2}} \Psi \Psi^{\prime} W_{2 z}^{(2)^{\prime \prime}}+24 \lambda_{z}^{t h^{2}} \Psi \Psi^{\prime} W_{2 z}^{(2)^{\prime \prime}}+24 \lambda_{z}^{t h^{2}} \Psi^{\prime} \Psi^{\prime \prime} W_{2 z}^{(2)^{\prime \prime}}+8 \lambda_{z}^{t h^{2}} W_{2 z}^{(2)^{\prime \prime} 2} \\
& -4\left(6\left(\lambda_{x}^{t h^{2}}-2 \lambda_{z}^{t h^{2}}\right) \Psi^{2} \Psi^{\prime}+12 \lambda_{z}^{t h^{2}} Y_{2 z}^{(2)}\left(\Psi-\Psi^{\prime \prime}\right)\right. \\
& +6 \lambda_{z}^{t h^{2}}\left(\Psi^{\prime}\left(-5 Y_{2 z}^{(2)^{\prime}}+\Psi^{\prime 2}+4 V_{2 z}^{(2)^{\prime}}+7 W_{2 z}^{(2)^{\prime}}\right)+2\left(2 V_{2 z}^{(2)}+3 W_{2 z}^{(2)}\right) \Psi^{\prime \prime}\right) \\
& \left.\left.+\lambda_{z}^{t h^{2}} \Psi\left(12 V_{2 z}^{(2)}+12 W_{2 z}^{(2)}-27 \Psi^{\prime} \Psi^{\prime \prime}+9 V_{2 z}^{(2)^{\prime \prime}}+16 W_{2 z}^{(2)^{\prime \prime}}\right)\right) \Psi^{\prime \prime \prime}+62 \lambda_{z}^{t h^{2}} \Psi^{2} \Psi^{\prime \prime \prime} 3\right)
\end{aligned}
$$

where the terms $\Psi, V_{2 z}^{(2)}, W_{2 z}^{(2)}$ and $Y_{2 z}^{(2)}$ are given by Eqs. (21), (66), (70), and (77), respectively.

\section{Discussion and conclusion}

This work aimed at investigating the nature of the elastic bifurcation undertaken by a growing soft layer subjected to an equi-biaxial strain. Considering a skin effect at the free surface, the instability thresholds are found to be controlled by a characteristic length, defined by the ratio between capillary energy and bulk elasticity. For the first time, a weakly nonlinear analysis of the wrinkling instability has been performed using the multiple-scale perturbation method applied to the incremental theory in finite elasticity. The Ginzburg-Landau equations are derived in Eq. (99) for different superposing linear modes: the resulting coefficients are collected in Table 1 for cylinders, squares and hexagonal patterns using two different values of the dimensionless ratio between the capillary length and the layer's thickness.

Considering the swelling of a soft gel as an illustrative example, recent experimental studies report a surface tension with the air at about $\gamma \simeq 43 \mathrm{mN} / \mathrm{m}$ (Tabuteau et al., 2009) with shear moduli ranging from few to hundreds of KPa (Trujillo et al., 2008), which give a characteristic capillary length $L_{c a p}$ in the order of the $\mu \mathrm{m}$. Since the typical layer's thickness $H$ is about a $\mathrm{mm}$, this gives a dimensionless ratio $L_{c a p} / H \simeq 10^{-3}$. The results of the weakly nonlinear coefficients in Table 1 reveal that all the bifurcation modes are subcritical. In practice, the wrinkled solutions are found to be highly unstable beyond the critical threshold, therefore the layer undergoes a discontinuous transition towards a state which will be selected by nonlinear effects of higher order. As depicted in Fig. 3, the weakly nonlinear elastic solutions tend to form sharp folds for relatively small amplitudes, suggesting a nonlinear transition towards a creased free surface. This finding is in accordance with the experimental results reporting a quick transition to creased states with significant hysteresis between onset and disappearance (Yoon et al., 2010). The theoretical predictions also suggest that the emergence of hexagonal patterns is energetically favored, as reported in early experiments (Tanaka et al., 1987). Nevertheless, although the wave amplitudes are considered all equal in this study, beyond the critical threshold further transitions may occur to states of lower symmetry for the hexagons.

On the contrary, the bifurcation becomes supercritical for higher capillary lengths, such as $L_{c a p} / H=0.025$, which means that wrinkles can be stabilized if the capillary length is of the same order of the layer's thickness, as usually found for fluid instabilities. Accordingly, it is reasonable to hypothesize that the formation of stable wrinkling in layered materials is driven by a competition of different elastic length-scales of the same order. 
In dynamic problems, the Ginzburg Landau equations provide further details on the post-bifurcation evolution of the selected patterns compared to existing static methods. In the proposed work, in fact, inertial effects allow both fixing the velocity of the near-critical elastic waves in Eq. (27) and deriving the correct scaling laws for the weakly nonlinear investigation in Eqs. (28) and (29). In the case of a supercritical bifurcation, i.e. a continuous transition from wrinkles to stable folds, it is noteworthy to highlight that the static amplitude solutions found here could be equivalently derived using the classical initial post-bifurcation analysis formulated by Koiter (1945). Although such a static method has been recently proved to capture the weakly nonlinear solution in a wide class of elastic problems (Cao and Hutchinson, 2012; Hutchinson, 2013), it must be reminded the dynamical analysis proposed here might provide further information on the post-buckling behavior after a subcritical, i.e. discontinuous, bifurcation. In this case, in fact, inertial effects cannot be neglected since they drive the transition towards a stable solution determined by fully nonlinear effects. The system dynamics has been found, for example, to have a strong influence on elastomer creasing, where sulci can nucleate in a finite time for an inertial or a gradient-descent dynamics but cannot get formed spontaneously for a viscoelastic material (see the Supplementary Material in Hohlfeld and Mahadevan, 2011).

Future work will be focused on investigating the effect of small imperfections within the system on pattern formation. In fact, the presence of material defects or small deviations from the perfectly equi-biaxial state could brake the symmetries by provoking different growth rates of the unstable modes, thus creating disordered patterns, as observed for Rayleigh-Bénard instability (Cross and Hohenberg, 1993).

In conclusion, the weakly nonlinear analysis presented in this study proves that soft layers subjected to an equi-biaxial strain undergo a pitchfork bifurcation driving the onset of the wrinkle-to-fold transition. In the case of growing gels, such a bifurcation is subcritical, and higher-order nonlinear interactions should be considered to investigate the formation of sharp folds at the free surface. Hexagonal patterns are found to be the most unstable modes in the weakly nonlinear regime near the instability threshold, albeit quasi-hexagonal patterns might later emerge because of an expected symmetry break. Finally, if the capillary length is of the same order of the layer's thickness, stable folds form with an amplitude scaling in the weakly nonlinear regime as the square root of the difference between the growth value and its linear stability threshold.

\section{Acknowledgments}

This work is in part supported by EU Grant ERG-256605, by the INSERM Grant OTPJ12U170 (Plan Cancer), and by the "Start-up Packages and PhD Program" project, co-funded by Regione Lombardia through the "Fondo per lo sviluppo e la coesione 2007-2013", formerly FAS program.

\section{Appendix A. Second order solution for a generic monochromatic mode}

Let me consider a monochromatic mode directed at an angle $\beta$ with respect to the principal direction $x$. The corresponding linear perturbation is defined as

$$
\begin{aligned}
& u_{x}^{(1)}=-\frac{\Psi^{\prime}(z)}{k} \cos \beta\left(A_{0}(\tau) e^{\imath k(x \cos \beta+y \sin \beta)}+\text { c.c. }\right) \\
& u_{y}^{(1)}=-\frac{\Psi^{\prime}(z)}{k} \sin \beta\left(A_{0}(\tau) e^{\imath k(x \cos \beta+y \sin \beta)}+\text { c.c. }\right) \\
& u_{z}^{(1)}=\Psi(z)\left(\imath A_{0}(\tau) e^{\imath k(x \cos \beta+y \sin \beta)}+\text { c.c. }\right)
\end{aligned}
$$

Considering all the interacting modes, the second order solution must be expressed as

$$
u_{i}^{(2)}=U_{i}^{(2)}(z)\left|A_{0}(\tau)\right|^{2}+\left(V_{i}^{(2)}(z) A_{0}^{2}(\tau) e^{2 l(x \cos \beta+y \sin \beta)}+\text { c.c. }\right)
$$

Accordingly, the solutions of Eqs. (34) and (35) with boundary conditions Eqs. (39) and (40) are given by

$$
V_{y}^{(2)}=\imath \sin \beta \frac{U_{z}^{(1), 2}-U_{z}^{(1)} U_{z}^{(1)^{\prime \prime}}+V_{z}^{(2) \prime}}{2 k} ; \quad V_{x}^{(2)}=\imath \cos \beta \frac{U_{z}^{(1) \prime 2}-U_{z}^{(1)} U_{z}^{(1)^{\prime \prime}}+V_{z}^{(2) \prime}}{2 k}
$$

while the other fields take the values previously calculated for the cylindrical mode in Eqs. (47) and (53). Such a result allows great simplifications when considering a superposition of linear waves, requiring that the second order solutions for the self-interacting modes have the same displacement fields.

\section{References}

Audoly, B., Boudaoud, A., 2008. Buckling of a stiff film bound to a compliant substrate-part ii: a global scenario for the formation of herringbone pattern J. Mech. Phys. Solids 56, 2422-2443.

Ben Amar, M., Ciarletta, P., 2010. Swelling instability of surface-attached gels as a model of soft tissue growth under geometric constraints. J. Mech. Phys. Solids 58, 935-954. 
Bender, C.M., Orszag, S.A., 1999. Advanced Mathematical Methods for Scientists and Engineers i: Asymptotic Methods and Perturbation Theory, vol. 1. Springer, New York.

Biot, M., 1963. Surface instability of rubber in compression. Appl. Sci. Res. 12, 168-182

Breid, D., Crosby, A.J., 2011. Effect of stress state on wrinkle morphology. Soft Matter 7, 4490-4496.

Cao, Y., Hutchinson, J.W., 2012. From wrinkles to creases in elastomers: the instability and imperfection-sensitivity of wrinkling. Proc. R. Soc. A $468,94-115$.

Ciarletta, P., 2013. Buckling instability in growing tumor spheroids. Phys. Rev. Lett. 110, 158102.

Cross, M.C., Hohenberg, P.C., 1993. Pattern formation outside of equilibrium. Rev. Mod. Phys. 65, 851.

Flavin, J., 1963. Surface waves in pre-stressed Mooney material. Q. J. Mech. Appl. Math. 16, 441-449.

Fu, Y., Ogden, R., 1999. Nonlinear stability analysis of pre-stressed elastic bodies. Continuum Mech. Thermodyn. 11, 141-172.

Fu, Y., Rogerson, G.A., 1994. A nonlinear analysis of instability of a pre-stressed incompressible elastic plate. Proc. R. Soc. Lond. A Math. Phys. Sci. 446, 233-254.

Golubitsky, M., Swift, J., Knobloch, E., 1984. Symmetries and pattern selection in Rayleigh-Bénard convection. Physica D 10, $249-276$.

Hohlfeld, E., Mahadevan, L., 2011. Unfolding the sulcus. Phys. Rev. Lett. 106, 105702.

Hong, W., Zhao, X., Suo, Z., 2009. Formation of creases on the surfaces of elastomers and gels. Appl. Phys. Lett. 95, 111901.

Hutchinson, J.W., 2013. The role of nonlinear substrate elasticity in the wrinkling of thin films. Philos. Trans. R. Soc. A $371,20120422$.

Kim, J., Yoon, J., Hayward, R.C., 2009. Dynamic display of biomolecular patterns through an elastic creasing instability of stimuli-responsive hydrogels. Nat Mater. 9, 159-164.

Kim, P., Abkarian, M., Stone, H.A., 2011. Hierarchical folding of elastic membranes under biaxial compressive stress. Nat. Mater. $10,952-957$.

Koiter, W.T., 1945. On the Stability of Elastic Equilibrium (in Dutch with English summary) (Thesis). Delft, H.J. Paris, Amsterdam.

Li, B., Cao, Y.-P., Feng, X.-Q., Gao, H., 2012. Mechanics of morphological instabilities and surface wrinkling in soft materials: a review. Soft Matter 8, 5728-5745.

Newell, A.C., Whitehead, J., 1969. Finite bandwidth, finite amplitude convection. J. Fluid Mech. 38, $279-303$.

Ogden, R.W., 1997. Non-Linear Elastic Deformations. Courier Dover Publications, New York.

Rodriguez, E.K., Hoger, A., McCulloch, A.D., 1994. Stress-dependent finite growth in soft elastic tissues. J. Biomech. 27, $455-467$.

Rogers, J.A., Someya, T., Huang, Y., 2010. Materials and mechanics for stretchable electronics. Science 327, $1603-1607$.

Saha, K., Kim, J., Irwin, E., Yoon, J., Momin, F., Trujillo, V., Schaffer, D.V., Healy, K.E., Hayward, R.C., 2010. Surface creasing instability of soft polyacrylamide cell culture substrates. Biophys. J. 99, L94-L96.

Tabuteau, H., Mora, S., Porte, G., Abkarian, M., Ligoure, C., 2009. Microscopic mechanisms of the brittleness of viscoelastic fluids. Phys. Rev. Lett. 102,

155501. Tanaka, H., Tomita, H., Takasu, A., Hayashi, T., Nishi, T., 1992. Morphological and kinetic evolution of surface patterns in gels during the swelling process:

evidence of dynamic pattern ordering. Phys. Rev. Lett. 68, 2794.

Tanaka, T., Sun, S.-T., Hirokawa, Y., Katayama, S., Kucera, J., Hirose, Y., Amiya, T., 1987. Mechanical instability of gels at the phase transition. Nature 325 , $796-798$.

Trujillo, V., Kim, J., Hayward, R.C., 2008. Creasing instability of surface-attached hydrogels. Soft Matter 4, $564-569$.

Yoon, J., Kim, J., Hayward, R.C., 2010. Nucleation, growth, and hysteresis of surface creases on swelled polymer gels. Soft Matter 6, 5807-5816. 